\title{
LXR nuclear receptors prompt a pro-inflammatory gene and functional profile in human macrophages
}

\section{Arturo González de la Aleja1}

Centro de Investigaciones Biológicas: Centro de Investigaciones Biologicas

\section{Mónica Torres-Torresano}

Hospital General Universitario Gregorio Maranon

Juan Vladimir de la Rosa

Universidad de las Palmas de Gran Canaria

\section{Barbara Alonso}

Centro de Investigaciones Biológicas: Centro de Investigaciones Biologicas

\section{Enrique Capa-Sardón}

Centro de Investigaciones Biológicas: Centro de Investigaciones Biologicas

\section{Amaya Puig-Kroger}

Hospital General Universitario Gregorio Maranon

\section{Miguel A Vega}

Centro de Investigaciones Biológicas: Centro de Investigaciones Biologicas

\section{Antonio Castrillo}

Instituto de Investigaciones Biomédicas Alberto Sols: Instituto de Investigaciones Biomedicas Alberto Sols

\section{Angel L. Corbi ( $\square$ acorbi@cib.csic.es )}

Consejo Superior de Investigaciones Científicas https://orcid.org/0000-0003-1980-5733

\section{Research Article}

Keywords: Inflammation, Innate immunity, Macrophage, Macrophage Polarization

Posted Date: February 15th, 2021

DOI: https://doi.org/10.21203/rs.3.rs-169946/v1

License: (c) (i) This work is licensed under a Creative Commons Attribution 4.0 International License. Read Full License 
1 LXR nuclear receptors prompt a pro-inflammatory gene and functional profile in human macrophages

4 Short title: LXR promotes a pro-inflammatory human macrophage signature

Arturo González de la Aleja ${ }^{1}$, Mónica Torres-Torresano ${ }^{2}$, Juan Vladimir de la Rosa ${ }^{3}$, Bárbara Alonso ${ }^{1}$,

7 Enrique Capa-Sardón ${ }^{1}$, Amaya Puig-Kröger ${ }^{2}$, Miguel A. Vega ${ }^{1}$, Antonio Castrillo ${ }^{3,4}{ }^{*}$, Ángel L. Corbí ${ }^{{ }^{*}}$

$9 \quad{ }^{1}$ Myeloid Cell Laboratory, Centro de Investigaciones Biológicas, CSIC, Madrid, Spain.

2 Unidad de Inmuno-Metabolismo, Instituto de Investigación Sanitaria Gregorio Marañón (liSGM),

11 Madrid, Spain.

3 Unidad de Biomedicina (Unidad Asociada al CSIC), Instituto Universitario de Investigaciones

Biomédicas y Sanitarias (IUIBS), Grupo de Investigación Medio Ambiente y Salud. Universidad de Las Palmas de Gran Canaria, Las Palmas, Spain.

${ }^{4}$ Instituto Investigaciones Biomédicas “Alberto Sols" (IIBM), and Centro Mixto Consejo Superior de Investigaciones Científicas y Universidad Autónoma de Madrid (ICSIC-UAM), Madrid, Spain.

* AC and ALC contributed equally to this work, and the order of authors is arbitrary.

20 Corresponding author: Dr. Ángel L. Corbí, Centro de Investigaciones Biológicas, CSIC. Ramiro de 21 Maeztu, 9. Madrid 28040; Phone: 34-91-8373112; FAX: 34-91-5627518; E-mail: acorbi@cib.csic.es ORCID ID: 0000-0003-1980-5733 
LXR promotes a pro-inflammatory human macrophage signature

23

24

25

26

27

28

29

30

31

32

\section{ABSTRACT}

Liver X Receptors (LXR) control cholesterol metabolism and exert anti-inflammatory actions in activated macrophages. However, their contribution to human macrophage polarization in the absence of pathogenic stimuli remains unclear. In fact, the LXR pathway has been reported to be significantly enriched in pro-inflammatory synovial macrophages from rheumatoid arthritis patients as well as in immunosuppressive tumors-associated macrophages from human metastatic colon tumors. To determine the role of LXR on macrophage differentiation and polarization, we have analyzed the contribution of LXR to the acquisition of the inflammatory and T-cell-activating functions of human monocyte-derived macrophages. We now report that LXR activation prompts the acquisition of a pro-inflammatory gene signature in human macrophages, whereas LXR inactivation results in enrichment of an anti-inflammatory gene profile. Accordingly, activation and inhibition of LXR oppositely alter the production of cytokines (e.g., TNF, IL1 $\beta$, CCL17, CCL19, IFN $\beta 1$ ) and T cell stimulation activities associated to human macrophage polarization. Mechanistically, the LXR-stimulated macrophage polarization shift relies on their ability to modulate the expression of MAFB and MAF, which govern the acquisition of the macrophage anti-inflammatory profile. The pathological significance of the LXR-mediated macrophage polarization shift was demonstrated by the ability of LXR agonists to modulate macrophage polarization promoted by either tumor-derived ascitic fluids or by synovial fluid from rheumatoid arthritis patients. As a whole, our results demonstrate that LXR activation prompts the acquisition of a pro-inflammatory transcriptional and functional specialization in human macrophages. 
LXR promotes a pro-inflammatory human macrophage signature

46

47

48

49

50

51

52

53

54

55

56

57

58

59

60

61

62

63

64

65

66

67

68

69

\section{INTRODUCTION}

Macrophages defend the organism against endogenous danger signals and exogenous threats, and initiate and resolve inflammatory responses. To perform these assorted tasks, macrophages can display a huge spectrum of activation ("polarization") states, whose acquisition depends on their developmental origin, tissue location, and prevailing extracellular cues (1-3). During inflammation, macrophages exert pro-inflammatory and anti-inflammatory/resolving effector functions, whose fine-tuning and sequential occurrence are crucial for tissue injury removal and return to homeostasis, and whose unbalance is associated to chronic inflammatory diseases.

M-CSF and GM-CSF have opposite instructing effects on macrophages during inflammatory responses $(4,5)$. M-CSF is indispensable for tissue-resident and monocyte-derived macrophage differentiation (6-9), and primes macrophages (M-M $\varnothing)$ for acquisition of an anti-inflammatory and immunosuppressive profile (IL10 high $\left.\operatorname{TNF}^{\text {low }} \operatorname{IL23^{\text {low}}} \operatorname{IL6}^{\text {low }}\right)(5,10-19)$. By contrast, GM-CSF is produced at sites of inflammation $(6,7)$, and primes macrophages $(G M-M \varnothing)$ for robust antigenpresenting, T cell-stimulatory and pro-inflammatory activity (IL10 ${ }^{\text {low }}$ TNF $^{\text {high }}$ IL23 ${ }^{\text {high }}$ IL6 $\left.{ }^{\text {high }}\right)$. Thus, M$\mathrm{M} \varnothing$ resemble tissue-resident 'trophic' macrophages, whereas GM-M $\varnothing$ represent pro-inflammatory monocyte-derived macrophages. In line with their opposite effector functions, M-M $\varnothing$ and GM-M $\varnothing$ exhibit distinct transcriptional profiles $(10,13,20-22)$ that resemble those of human resident and inflammatory macrophages in vivo (22-25), and also differ in their responsiveness to methotrexate (MTX) $(26,27)$.

LXR $\alpha$ and LXRß (coded for by $N R 1 H 3$ and $N R 1 H 2$, respectively) are ligand-activated transcription factors that regulate gene expression (positively and negatively) in a ligand-dependent manner, and that actively control macrophage differentiation and specialization $(28,29)$. LXR activity is crucially 
LXR promotes a pro-inflammatory human macrophage signature

involved in cellular cholesterol metabolism in most tissues. Upon binding of ligand (endogenous cholesterol derivatives, or synthetic agonists like T0901317 and GW3965), LXR positively control the expression of genes that collectively inhibit uptake and promote cholesterol efflux, thus contributing to prevent cellular lipid overload. LXR ligands also control inflammation in macrophages by antagonizing the induction of inflammation-related genes after activation $(29,30)$. These results have led to recognition of LXR as anti-atherogenic and anti-inflammatory factors (31, 32). However, LXR activation exacerbates inflammatory responses in human monocytes, dendritic cells and in a mouse arthritis model (33-36), and agonist-induced LXR activation elicits anti-tumor activity through immune-mediated mechanisms (37), specifically reducing the abundance of immunosuppressive Myeloid-Derived Suppressor Cells (MDSC) and enhancing anti-tumoral cytotoxic T lymphocytes (CTLS) activation (37). Of note, the LXR pathway is highly upregulated in pro-inflammatory macrophages from rheumatoid arthritis (RA) synovial fluid (RASF-M $\varnothing$ ), where LXR potentiate TLR-driven cytokine release (38), but is also the most enriched pathway in large tumorassociated macrophages (large TAM) (39), which are immunosuppressive and correlate with a worse prognosis (40). This apparently discrepant findings illustrate the ambiguous contribution of LXR to inflammatory and immune responses, and raises the question of the role of LXR factors to human macrophage polarization $(33,41)$.

To address this issue, we have now determined the range of LXR target genes in anti-inflammatory M-M $\varnothing$ and pro-inflammatory $\operatorname{GM}-\mathrm{M} \varnothing(13,22)$, and assessed the contribution of LXR to the acquisition of the transcriptional and functional signature of human macrophages through the use of specific LXR agonist (GW3965) and inverse agonist (GSK2033). We report that LXR controls human macrophage specialization, and that LXR activation primes monocytes towards the acquisition of the transcriptional and functional properties of pro-inflammatory macrophages. 
LXR promotes a pro-inflammatory human macrophage signature

94

95

96

97

98

99

100

101

102

103

104

105

106

107

108

109

110

111

112

113

114

\section{EXPERIMENTAL PROCEDURES}

Generation of human monocyte-derived macrophages in vitro and treatments. Human Peripheral Blood Mononuclear Cells (PBMCs) were isolated from buffy coats from anonymous healthy donors over a Lymphoprep (Nycomed Pharma) gradient according to standard procedures. Monocytes were purified from PBMC by magnetic cell sorting using anti-CD14 microbeads (Miltenyi Biotec). Monocytes ( $>95 \% \mathrm{CD} 14^{+}$cells) were cultured at $0.5 \times 10^{6}$ cells $/ \mathrm{ml}$ in Roswell Park Memorial Institute (RPMI 1640, Gibco) medium supplemented with 10\% fetal bovine serum (FBS, Biowest) for 7 days in the presence of $1000 \mathrm{U} / \mathrm{ml} \mathrm{GM-CSF}$ or $10 \mathrm{ng} / \mathrm{ml} \mathrm{M-CSF}$ (ImmunoTools) to generate GM-CSFpolarized macrophages (GM-M $\varnothing$ ) or M-CSF-polarized macrophages (M-M $\varnothing$ ), respectively (42). Cytokines were added every two days and cells were maintained at $37^{\circ} \mathrm{C}$ in a humidified atmosphere with $5 \% \mathrm{CO} 2$ and $21 \% 02$. Where indicated, macrophages were treated at different time points with one dose of LXR agonist GW3965 (1 $\mu \mathrm{M}$, Tocris), LXR inverse agonist GSK2033 (1 $\mu \mathrm{M}$, Tocris) or both, using dimethyl sulfoxide (DMSO) as vehicle. In the dual condition, the inverse agonist was added 1hour prior to agonist treatment. When indicated, either Synovial Fluid from Rheumatoid Arthritis (RASF) patients or Ascitic Fluid from cancer patients (AF) was added to monocytes (0.2:1 in culture medium for RASF, $0.5: 1$ in culture medium for AF), and the cultures were maintained for $72 \mathrm{~h}$. Synovial fluid samples were obtained by therapeutic arthrocentesis from the knee joints of patients with active Rheumatoid Arthritis (24). Ascitic fluids from metastatic tumors were kindly provided by Dr. Mạ. Isabel Palomero (Oncology Department, Hospital General Universitario Gregorio Marañón). Samples were centrifuged (4000xg, $15 \mathrm{~min}$ ) to remove cells and particulate material, sterile-filtered, aliquoted, and stored at $-20^{\circ} \mathrm{C}$ until use. The patients provided informed consent and the Hospital General Universitario Gregorio Marañón ethics committee approved the study. For macrophage activation, cells were treated with $10 \mathrm{ng} / \mathrm{ml}$ E. coli 055:B5 lipopolysaccharide (Ultrapure LPS, SigmaAldrich). Human cytokine production was measured in supernatants collected from treated M-M $\varnothing$ 
and GM-M $\varnothing$ using commercial ELISA [(TNF- $\alpha$, CCL2 (BD Biosciences), IL-10, IL-6 (Biolegend), Activin A, CCL19, CCL17, IL1 $\beta$, IFN $\beta 1$ (R\&D Systems) and MMP12 (Bosterbio)] according to the procedures supplied by the manufacturers.

Quantitative real-time RT-PCR (qRT-PCR). Total RNA was extracted using the total RNA and protein isolation kit (Macherey-Nagel). RNA samples were reverse-transcribed with High-Capacity cDNA Reverse Transcription reagents kit (Applied Biosystems) according to the manufacturer's protocol. Real-time quantitative PCR was performed with LightCycler ${ }^{\circledR} 480$ Probes Master (Roche Life Sciences) and Taqman probes on a standard plate in a Light Cycler ${ }^{\circledR} 480$ instrument (Roche Diagnostics). Gene-specific oligonucleotides (Supplementary Table 1) were designed using the Universal ProbeLibrary software (Roche Life Sciences). Results were normalized to the expression level of the endogenous references genes TBP, HPRT1 and GAPDH and quantified using the $\triangle \triangle C T$ (cycle threshold) method.

Western blot. M-M $\varnothing$ and GM-M $\varnothing$ cell lysates were subjected to SDS-PAGE (50 $\mu \mathrm{g}$ unless indicated otherwise) and transferred onto an Immobilon-P polyvinylidene difluoride membrane (PVDF; Millipore). After blocking the unoccupied sites with 5\% non-fat milk diluted in Tris-Buffered Saline plus Tween 20 (TBS-T), protein detection was carried out with antibodies against LXR $\alpha$ (PPZ0412; Biotechne), LXRß (PPK8917; Biotechne), MAFB (Sigma Aldrich) or c-MAF (sc-7866; Santa Cruz Biotechnology). Protein loading was normalized using an antibody against GAPDH (sc-32233; Santa Cruz Biotechnology) or vinculin (V9131; Sigma-Aldrich). Quimioluminiscence was detected in a Chemidoc Imaging system (BioRad) using SuperSignal ${ }^{\mathrm{TM}}$ West Femto (ThermoFisher Scientific). 
LXR promotes a pro-inflammatory human macrophage signature

141 Small Interfering Ribonucleic Acid (siRNA) Transfection. M-M $\varnothing$ or GM-M $\varnothing\left(1 \times 10^{6}\right.$ cells) were 142 transfected with human NR1H3-specific siRNA (siNR1H3, $50 \mathrm{nM}$ ) (s19568, Thermo-Fisher Scientific) 143 and/or human NR1H2-specific siRNA (siNR1H2, 50nM) (s14684; Thermo-Fisher Scientific) using 144 HiPerFect (Qiagen). Silencer ${ }^{\mathrm{TM}}$ Select Negative Control No. 1 siRNA (siCtrl, 50 nM) (\#4390843; 145 Thermo-Fisher Scientific) was used as negative control siRNA. Six hours after transfection, cells were 146 allowed to recover from transfection in complete medium (18 $\mathrm{h}$ ) and then lysed. Knock-down of $147 \mathrm{NR} 1 \mathrm{H} 3 / \mathrm{LXR} \alpha$ or NR1H2/LXR $\beta$ was confirmed by q-PCR and western blot. Monocytes $\left(1 \times 10^{6}\right.$ cells $)$ 148 were transfected with human MAFB-specific smart Pool (siMAFB, 25 nM) (M-009018; Dharmacon) 149 using HiPerFect (Qiagen). siGenome non-targeting siRNA Pool\#2 (siCtrl, 25 nM) (D-001206; 150 Dharmacon) was used as negative control siRNA. Four hours after transfection, cells were treated 151 with GSK2033 (1 $\mu \mathrm{M}$, Tocris) and allowed to recover from transfection in complete medium for 36 $152 \mathrm{~h}$ before lysis.

154 Mixed Leukocyte Reaction (MLR). Untreated or treated M-M $\varnothing$ and GM-M $\varnothing$ were detached using 155 Trypsin-EDTA (Gibco) at 37으, and re-plated in a 96 -well U-bottom plates ( $10^{4}$ cells/well) in RPMI 156 with $5 \%$ human AB serum (Lonza) for 24 hours. Allogeneic T lymphocytes were isolated from PBMCs 157 using CD3+ magnetic beads (Miltenyi Biotec), and co-cultured with macrophages (10:1 T 158 lymphocyte:macrophage ratio) for 6 days in RPMI with $5 \%$ human AB serum. Then, ${ }^{3} \mathrm{H}$-Thymidine (1 $159 \mu \mathrm{Ci} /$ well, Perkin Elmer) was added and, after 18 hours, radioactivity was transferred to a filter and 160 thymidine counts measured in a scintillation counter (Perkin Elmer).

RNA-sequencing and data analysis. RNA was isolated from GM-M $\varnothing$ or M-M $\varnothing$ generated from 163 monocytes exposed to a single dose of DMSO, GW3965, GSK2033 or both at the beginning of the 164 differentiation process, and subjected to sequencing on a BGISEQ-500 platform 
LXR promotes a pro-inflammatory human macrophage signature

165 (https://www.bgitechsolutions.com). RNAseq data were deposited in the Gene Expression Omnibus 166 (http://www.ncbi.nlm.nih.gov/geo/) under accession GSE156783 and GSE156696. On average, $16788.04 \mathrm{M}$ reads per sample were generated and clean reads were mapped to the reference (UCSC 168 Genome assembly hg38) using Bowtie2 (average mapping ratio to reference genome, 91.82\%) (43). 169 Gene expression levels were calculated by using the RSEM software package (44), and differential 170 gene expression was assessed by using the R-package DESeq2 algorithms using the parameters Fold 171 Change $>2$ and adjusted $p$ value $<0.05$. Plots generated with the ggplot 2 package, and heatmaps and 172 clustering were done using the Genesis software (http://genome.tugraz.at/genesisclient/) (45). 173 Differentially expressed genes were analysed for annotated gene sets enrichment using ENRICHR 174 (http://amp.pharm.mssm.edu/Enrichr/) (46, 47), and PANTHER (48), and enrichment terms 175 considered significant with a Benjamini-Hochberg-adjusted $p$ value $<0.05$. For gene set enrichment 176 analysis (GSEA) (http://software.broadinstitute.org/gsea/index.jsp) (49), gene sets available at the 177 website, as well as gene sets generated from publicly available transcriptional studies 178 (https://www.ncbi.nlm.nih.gov/gds), were used. Detection of LXR-binding elements was done on 179 the available ChIP-Seq data from GW3965-treated colorectal adenocarcinoma HT29 cells (GEO 180 GSM2042848) (50) using the Cistrome Data Browser (http://cistrome.org/db/\#/) and the DMINDA2 181 (regulatory DNA motif identification and analysis) web server 182 (https://bmbl.bmi.osumc.edu/DMINDA2/index.php) (51).

Statistical analysis. For comparison of means, and unless otherwise indicated, statistical significance 185 of the generated data was evaluated using the paired Student $t$ test in GraphPad Prism 8. In all cases, 186 $\mathrm{p}<0.05$ was considered as statistically significant. 
LXR promotes a pro-inflammatory human macrophage signature

187

188

189

190

191

\section{RESULTS}

Differential expression of LXR $\alpha$ and LXR-regulated genes in GM-M $\varnothing$ and $M-M \emptyset$. We have previously defined two gene sets whose expression marks human monocyte-derived proinflammatory GM-M $\varnothing$ ("Pro-inflammatory gene set") and anti-inflammatory M-M $\varnothing$ ("Antiinflammatory gene set") (Figure $1 \mathrm{~A})(13,22)$, and also discriminates the gene profiles of proinflammatory and immunosuppressive macrophages in vivo (24). Accordingly, the GM-M $\varnothing$ transcriptome exhibits an over-representation of genes highly expressed by rheumatoid arthritis synovial fluid macrophages (RASF-M $\varnothing$ ) (52), whereas the transcriptome of M-M $\varnothing$ shows a strong enrichment of genes preferentially expressed by tumor-associated macrophages (TAM) with significant prognostic relevance (39) (Figure 1B). Remarkably, however, the LXR pathway has been reported to be highly upregulated in both pro-inflammatory RASF-M $\varnothing(38)$ and immunosuppressive TAM from colorectal liver metastasis (39), thus raising the question of the role of LXR on human macrophage polarization. To clarify this apparent discrepancy, we sought to determine the role of LXR along the differentiation of both pro-inflammatory GM-M $\varnothing$ and anti-inflammatory M-M $\varnothing$.

Protein analysis on a large number of independent macrophage samples revealed a higher expression of LXR $\alpha$ (encoded by NR1H3) in GM-M $\varnothing$, and a higher level of NR1H2-encoded LXR $\beta$ in $\mathrm{M}-\mathrm{M} \varnothing$ (Figure 1C-D), a result that agrees with the relative expression of $N R 1 H 3$ and NR1H2 mRNA in both macrophage subtypes (Figure 1E). Regarding LXR target genes [defined by positive responsiveness to desmosterol, (53)], their expression was not associated to a specific macrophage subtype (Figure 1F) and, in fact, GSEA revealed that the expression of LXR-target genes diverged between M-M $\varnothing$ and GM-M $\varnothing$ (data not shown). However, and unlike $L P L$ (54) and APOE (55), the LXR target genes ABCA1 (56), ABCG4 (57), MERTK (58), ARL4C (59), ARG2 (60) and PLTP (61) were 
LXR promotes a pro-inflammatory human macrophage signature

210

211

212

213

214

215

216

217

218

219

220

221

222

223

224

225

226

227

228

229

230

expressed at higher levels in anti-inflammatory $M-M \varnothing$ (Figure 1G), thus suggesting that the extent of expression of classical LXR targets tends to be higher in M-M $\varnothing$.

LXR activation shifts the M-CSF-dependent differentiation of M-M $\varnothing$ towards the proinflammatory side. To address the extent of the LXR dependency of the transcriptome of M-M $\varnothing$, monocytes were exposed to a single dose of either the LXR agonist GW3965 (1 $\mu M$ ), the LXR inverse agonist GSK2033 (1 $\mu \mathrm{M}$ ) or both (GSK2033 1 hour prior to GW3965) at the beginning of the differentiation process with M-CSF, and the transcriptome of the resulting macrophages was determined (Figure 2A). As expected, GW3965 treatment enhanced the expression of LXRdependent genes (53), an effect that was abrogated or impaired by GSK2033 (Figure 2B). Exposure to GW3965, and to a lower extent GSK2033, notably altered the transcriptome of M-M $\varnothing$ (Figure $2 C)$. Indeed, expression of the M-M $\varnothing$-specific and GM-M $\varnothing$-specific gene sets were very significantly altered in GW3965-M-M $\varnothing$ and GSK2033-M-M $\varnothing$ (Figure 2D), an effect also evident upon clustering of the genes modulated by either ligand (Figure 2E, F). The most unambiguous effect was observed in the case of GW3965-M-M $\varnothing$, where the expression of the M-M $\varnothing$-specific and GM-M $\varnothing$-specific gene sets was dramatically enhanced and impaired, respectively (Figure 2D), while expression of the GM-M $\varnothing$-specific gene set was reduced in GSK2033-M-M $\varnothing$ (Figure 2D). Of note, GW3965 treatment impaired the expression of genes associated to the anti-inflammatory activities of M-M $\varnothing$ (IL10, FOLR2, CD209) $(22,62,63)$, and augmented the expression of paradigmatic genes of the GM-M $\varnothing$ specific gene sets (CD226, EGLN3) (Figure 2D, G). Therefore, LXR activation favors the acquisition of a more pro-inflammatory transcriptional profile in anti-inflammatory $M-M \emptyset$. Interestingly, the proinflammatory effect of GW3965 varied along M-M $\varnothing$ differentiation, as its inhibitory effect on the expression of the M-M $\varnothing$-specific gene set (e.g., IGF1, FOLR2, HTR2B, CD163L1, IL10, CCL2) was more profound at the beginning of the differentiation process (Figure $3 \mathrm{~A}, \mathrm{~B}$ ), and was weaker at later time 
LXR promotes a pro-inflammatory human macrophage signature

234 points (Figure 3A). By contrast, the inverse agonist GSK2033 had a negligible effect by itself, 235 although it completely prevented the effect of the agonist (Figure 3A, B). Therefore, the pro236 inflammatory consequences of LXR activation are maximal at the start of the M-M $\varnothing$ differentiation 237 process and gradually weakens at later time points.

239 The functional significance of the LXR-dependent transcriptional changes in M-M $\varnothing$ was weighed by 240 comparing the cytokine profiles and the allogeneic T lymphocyte-activating ability of $M-M \varnothing$, GW3965-M-M $\varnothing$ and GSK2033-M-M $\varnothing$. Compared to M-M $\varnothing$, GW3965-M-M $\varnothing$ produced higher levels of IL-1 $\beta$ and the immuno-stimulatory CCL19 chemokine, but lower levels of the anti-inflammatory cytokine IL-10 (Figure 3C). This trend towards a more pro-inflammatory cytokine profile was also observed upon LPS stimulation, as GW3965-M-M $\varnothing$ secreted higher levels of TNF, IFN $\beta 1$ and IL1 $\beta$ (Figure 3D). Since the pro-inflammatory effect of GW3965 treatment was impaired or abolished in the presence of GSK2033 (Figure 3A, B), these results demonstrate that LXR activation prompts the acquisition of a more pro-inflammatory transcriptional and cytokine profile in anti-inflammatory MM $\varnothing$. Regarding immunogenic ability, GSK2033-M-M $\varnothing$ and GW3965-M-M $\varnothing$ triggered a weaker allogenic T cell proliferation than control $M-M \varnothing$, an effect that was more significant and consistent in the case of GSK2033-M-M $\varnothing$ (Figure 3E). Therefore, modulation of LXR activity impacts the inflammatory and immunogenic activity of $M-M \varnothing$, with LXR activation promoting the acquisition of a more pro-inflammatory phenotypic (transcriptional) and functional profile.

LXR inhibition modulates the GM-CSF-dependent macrophage differentiation towards the antiinflammatory side. To assess the extent of the above findings, LXR activity was also pharmacologically modulated prior to the GM-CSF-driven differentiation of pro-inflammatory GM- 
LXR promotes a pro-inflammatory human macrophage signature

258

whereas GSK2033 had the opposite effect (Figure 4B), and the transcriptome of GW3965-GM-M $\varnothing$ and GSK2033-GM-M $\varnothing$ were significantly distinct from that of control GM-M $\varnothing$ (Figure 4C). Indeed, GSK2033-GM-M $\varnothing$ displayed higher levels of the anti-inflammatory $M-M \emptyset$-specific gene set and diminished expression of the pro-inflammatory GM-M $\varnothing$-specific gene set (Figure 4D). The changes observed in GW3965-GM-M $\varnothing$ were less significant (Figure 4D), as also observed after clustering of the genes modulated by either LXR ligand, thus indicating that GM-M $\varnothing$ differentiation is more sensitive to the action of the inverse agonist GSK2033, that enhances the expression of genes of the anti-inflammatory M-M $\varnothing$-specific gene set and limits the acquisition of the pro-inflammatory GM$\mathrm{M} \emptyset$-specific gene set (Figure 4E-G). These conclusions were supported by gene ontology analysis using PROFILER and GSEA, that revealed a significant enrichment of the "HALLMARK_INFLAMMATORY_RESPONSE" and "HALLMARK_TNFA_SIGNALING_VIA_NFKB" gene sets in GW3965-GM-M $\varnothing$, as well as a negative enrichment of the "HALLMARK_INFLAMMATORY_RESPONSE" gene set in GSK2033-GM-M $\varnothing$ (Supplementary Figure 1A, B). Significantly, analysis of ChIP-Seq public datasets (GEO GSM2042848) identified functional LXR-binding elements (50) in the vicinity of pro-inflammatory genes upregulated by GW3965 in both GM-M $\varnothing$ and M-M $\varnothing$ (Supplementary Figure 2). Taken together, these results obtained on GM-M $\varnothing$ and M-M $\varnothing$ demonstrated that GW3965-activated LXR promotes the acquisition of a more proinflammatory macrophage profile, an effect limited by GSK2033 that, by contrast, enhances the antiinflammatory specialization of monocyte-derived macrophages (that is, the acquisition of the antiinflammatory M-M $\varnothing$-specific gene set).

The strongest transcriptional effects of GSK2033 were observed at early stages of the differentiation of $\mathrm{GM}-\mathrm{M} \varnothing$, a time at which the inverse agonist maximally impaired the expression of the GM-M $\varnothing$ specific gene set and promoted the acquisition of genes of the $M-M \varnothing$-specific gene set (Figure 5A, 

limited to 24-48 hours (Supplementary Figure 3A-C), and modulation of LXR activity for short time periods significant changed the expression of $G M-M \emptyset$-specific genes when using lower concentrations of GM-CSF (Supplementary Figure 3D). These results, therefore, suggest that LXR influences the expression of genes whose expression reflects the pro-inflammatory or antiinflammatory specialization of monocyte-derived human macrophages. Therefore, like in the case of $M-M \varnothing$, the transcriptional consequences of LXR activity modulation are more profound at the start of the GM-M $\varnothing$ differentiation process.

LXR activity shapes the inflammatory and immuno-stimulatory functions of GM-M $\varnothing$. To evaluate the influence of LXR activity on GM-M $\varnothing$ effector functions, we first determined the cytokine profile of GW3965-GM-M $\varnothing$ and GSK2033-GM-M $\varnothing$. Compared to GM-M $\varnothing$ or GW3965-GM-M $\varnothing$, GSK2033GM-M $\varnothing$ produced significant lower levels of IL-1 $\beta$ and the pro-inflammatory chemokine CCL17

(Figure 5C), as well as lower levels of TNF, IL-10, CCL8, CCL19, IL-1 $\beta$ and IFN $\beta 1$ in response to LPS

(Figure 5D, E), in line with the anti-inflammatory effect of LXR inhibition. Conversely, GW3965-GM-

$M \varnothing$ produced higher levels of TNF, IL-10, CCL19, IL-1 $\beta$ and IFN $\beta 1$ after LPS stimulation, as well as an elevated TNF/IL10 ratio (Figure 5D, E). As a whole, this set of data indicates that the acquisition of the pro-inflammatory cytokine profile that characterizes GM-M $\varnothing$ is favored by LXR activation and impaired by LXR inhibition, what agrees with the respective transcriptional effects of GW3965 and GSK2033.

Regarding T cell activation, GW3965-GM-M $\varnothing$ showed higher allogeneic T lymphocyte-activating activity than untreated $\mathrm{GM}-\mathrm{M} \varnothing$ (Figure $5 \mathrm{~F}, \mathrm{G}$ ), an increase that correlated with their higher expression of $C D 80$ (Figure $5 \mathrm{H}$ ), which encodes a critical T cell co-stimulatory molecule. Therefore, 
306

and like in the case of $M-M \emptyset$, the level of activation of LXR determines the acquisition of T cellstimulatory ability during GM-M $\varnothing$ differentiation. Indeed, the enhanced T cell stimulatory activity of GW3965-GM-M $\varnothing$ was prevented by the inverse agonist GSK2033 (compare GW3965-GM-M $\varnothing$ with GW/GSK GM-M $\varnothing$, Figure 5F,G). Therefore, and in agreement with the transcriptional data, GW3965-dependent LXR activation favors the acquisition of GM-M $\varnothing$-specific functions, an effect prevented by the GSK2033-mediated LXR inhibition.

\section{Molecular mechanisms underlying the macrophage programming effect of LXR: Role of MAFB and}

Activin A. While MAFB and MAF are master regulators for the differentiation of anti-inflammatory $M-M \varnothing(42,64,65)$, the GM-CSF-driven generation of $G M-M \varnothing$ is dependent on activin $A(13,22)$. Given the involvement of LXR factors on the acquisition of the transcriptional and functional profile of GM-M $\varnothing$ and M-M $\varnothing$, we hypothesized that LXR might alter the relative levels of MAF/MAFB or activin A. The first hypothesis was supported by the significantly higher expression of MAFBdependent genes in GSK2033-GM-M $\varnothing$ (Figure 6A). In fact, Western blot revealed that, compared to their corresponding controls, GSK2033-treated macrophages (GSK2033-GM-M $\varnothing$ and GSK2033-M$M \varnothing$ ) expressed higher levels of both MAFB and MAF (Figure 6B). Since GW3965-M-M $\varnothing$ exhibited reduced levels of MAF than M-M $\varnothing$ (Figure 6B), these results indicate that the influence of LXR on the generation of GM-M $\varnothing$ and M-M $\varnothing$ correlates with a distorted expression of MAFB and, to a lower extent, MAF. Conversely, although GW3965-M-M $\varnothing$ produced slightly higher levels of activin A than control M-M $\varnothing$ (Figure 6C), and GSK2033-GM-M $\varnothing$ exhibited lower activin A production (Figure 6C), the association of activin A production with the skewed generation of GM-M $\varnothing$ and M-M $\varnothing$ was not straightforward. 
LXR promotes a pro-inflammatory human macrophage signature

329 The implication of the enhanced expression of MAFB in the effect of GSK2033 was assessed by 330 determining the expression of MAFB and MAFB targets in differentiating monocytes exposed to 331 GSK2033 after siRNA-mediated silencing of MAFB (Figure 6D). In this experimental set-up, GSK2033 332 increased the expression of MAFB and the MAFB target $L G M N$, and this enhancing effect was 333 inhibited when MAFB levels were knocked-down (Figure 6E). Conversely, MAFB levels did not 334 significantly modified the ability of GSK2033 to blunt ABCA1 expression (Figure 6E). Therefore, the 335 effect of the LXR inverse agonist GSK2033 on monocyte-to-macrophage differentiation is mediated, 336 at least partly, through the augmented expression of MAFB.

Modulation of macrophage specialization by LXR: physio-pathological relevance. Since the LXR pathway is highly upregulated in pro-inflammatory macrophages from rheumatoid arthritis (RA) synovial fluid (RASF-M $\varnothing$ ) (38), and is also the most enriched pathway in immunosuppressive tumorassociated macrophages (39), we sought to analyze the apparently ambiguous contribution of LXR to macrophage polarization. To that end, we first evaluated whether LXR is involved in the macrophage polarizing ability of tumor-conditioned fluids, and specifically of ascitic fluids from tumors of various origins (Figure 7A). The M-CSF-induced expression of two functionally relevant markers of tumor-associated macrophages, IGF-1 $(66,67)$ and FOLR2 $(63,68)$, was prevented in the presence of the LXR agonist GW3965 (Figure 7B). Moreover, the upregulation of both genes in the presence of ascitic fluids was also impaired by the LXR agonist (Figure 7B), implying that the polarizing ability of tumor-conditioned media is dependent on LXR activity. Next, we evaluated the contribution of LXR to the polarizing ability of RA synovial fluid (Figure 7C). As shown in Figure 7D, all analyzed RA synovial fluids potentiated the expression of NR1H3 and LXR targets (ABCA1), an

351 effect completely abrogated by the LXR inverse agonist GSK2033. More importantly, RA synovial 352 fluids also augmented, albeit variably, the expression of the GM-M $\varnothing$-specific genes $C D 1 B$ and 
LXR promotes a pro-inflammatory human macrophage signature

353 INHBA, whose enhancement was also prevented by GSK2033 (Figure 7D), indicating that LXR also 354 directly contributes to the pro-inflammatory polarization effect of RA synovial fluids. Altogether, 355 these results evidenced that LXR mediates the effect of two biological fluids (synovial and ascitic) 356 that promote the acquisition of opposite functional states in human macrophages, thus emphasizing 357 the critical role of LXR in human macrophage polarization. 
LXR promotes a pro-inflammatory human macrophage signature

358

359

360

361

362

363

364

365

\section{DISCUSSION}

LXR nuclear receptors have a prominent role in lipid and cholesterol homeostasis, but also regulate the expression of inflammatory mediators [reviewed in (69)] and are absolutely required for the generation of specialized splenic macrophages (70). To clarify the role of LXR in human innate cells, we have analyzed the role of LXR activation in macrophage differentiation and polarization, primarily through the use of pharmacological approaches. Our results indicate that LXR has a remarkable influence on the acquisition of the transcriptome that defines pro-inflammatory (GMCSF-dependent) and anti-inflammatory (M-CSF-dependent) monocyte-derived macrophages, and that LXR over-activation promotes the acquisition of a pro-inflammatory transcriptional and functional profile, whereas abrogation of LXR activity favors the acquisition of anti-inflammatory capacities. Indeed, LXR modulates the cytokine profile of macrophages and modifies their T cell activation activity, a result that, to our knowledge, has not been previously reported. Mechanistically, modulation of LXR activity led to diminished levels of the factors that determine the pro- and anti-inflammatory polarization of human macrophages, namely activin $A$ and MAF/MAFB, respectively. As a whole, our findings reveal a net pro-inflammatory effect of LXR during macrophage differentiation in the presence of either GM-CSF or M-CSF. The pathological relevance of our findings is illustrated by the LXR-dependency of the macrophage-polarizing action of both synovial fluid from Rheumatoid Arthritis patients (pro-inflammatory) and tumor-derived ascitic fluids (immunosuppressive).

Numerous studies have evidenced that LXR suppress inflammation in the presence of pathogenic stimuli through direct and indirect mechanisms, including transactivation of anti-inflammatory genes and trans-repression of NFKB- and AP1-dependent genes, and in vivo results have confirmed the net anti-inflammatory effects of LXR in the presence of macrophage-stimulating agents 
LXR promotes a pro-inflammatory human macrophage signature

382 [reviewed in (69)]. The pro-inflammatory action of LXR that we now report is compatible with those 383 previous studies because we have assayed the effect of LXR activation on unstimulated monocytes 384 that were subsequently exposed to either GM-CSF or M-CSF. Indeed, it is worth noting that the pro385 inflammatory actions of LXR are not unprecedented and have been previously noted (30). Leaving 386 aside the positive correlation between LXR activation and Rheumatoid Arthritis $(34,35,38)$, long 387 term pre-treatment of primary human macrophages to LXR ligands results in potentiated LPS 388 responses (33), and LXR activation leads to increase dendritic cell maturation at the phenotypic, 389 cytokine and functional levels (36). Further, LXR agonists have been recently reported to trigger 390 trained immunity in human monocytes, whereby LXR activation primes macrophages for enhanced 391 responses towards secondary stimuli (41) and impairs MDSC-mediated immunosuppression in 392 cancer (37). While these findings are in line with the enhanced LPS-induced TNF production by 393 GW3965-M-M $\varnothing$ (Figure 2), they can also be interpreted as LXR agonist having a macrophage re394 polarizing action, because differentiation of $M-M \varnothing$ in the presence of GW3965 results in 395 macrophages with an GM-M $\varnothing$-like transcriptional and functional profile (enhanced expression of 396 the "Pro-inflammatory gene set", diminished levels of MAF/MAFB, impaired basal IL-10 production). 397 However, and regardless of the interpretation, all these results illustrate the ability of LXR for 398 macrophage pro-inflammatory re-programming, and suggest that LXR activity modulates 399 inflammatory responses in a cell- or activation-dependent manner, a suggestion that is reminiscent 400 of the macrophage-specific effects of the LXR ligand desmosterol (53).

402 In the context of the net pro-inflammatory effect of LXR during human monocyte-to-macrophage 403 differentiation, the macrophage re-polarizing action of LXR also shows a high degree of cell404 specificity, as the sensitivity to LXR ligands greatly differ among monocytes, differentiating and fully 405 differentiated M-M $\varnothing$ or GM-M $\varnothing$. Globally, the effect of LXR activity modulators on the expression 
LXR promotes a pro-inflammatory human macrophage signature

406

407

408

409

410

411

412

413

One possible mechanism underlying the effect of LXR on macrophage pro-inflammatory polarization

421 can be interpreted by our pharmacological analysis. Inhibition of LXR was found to rise the 422 acquisition of an anti-inflammatory transcriptome and led to a significant increase in the expression 423 of MAF and MAFB, both of which determine the anti-inflammatory polarization of macrophages and 424 the production of IL-10 $(42,65,71,72)$. Whether LXR directly controls the expression of MAF/MAFB 425 in human macrophages would need further investigation. One possible clue came to our attention 426 through the analysis of available datasets from other cell lineages, which confirm the presence of 427 functional LXR-binding sites within GM-M $\varnothing$-specific genes ("Pro-inflammatory gene set) that are 428 specifically upregulated in the presence of GW3965 (http://cistrome.org/db/\#/; CistromeDB: 429

of polarization-specific genes diminishes along monocyte-to-macrophage differentiation and is inversely correlated with the acquisition of functional polarization. In fact, the sensitivity to LXR modulation at later stages of differentiation is only observed when low doses of priming cytokines are used. Therefore, the impact of LXR activity on macrophage polarization and functions is more relevant at the monocyte stage and diminishes along macrophage differentiation. The more prominent action of LXR at the beginning of the differentiation model is compatible with a sequential and ordered involvement of different factors, including lineage-determining factors and signal-dependent factors. In our opinion, the potent ligand-activation effect on LXR activity before the growth factor trigger, unbalances the polarization towards a sustained pro-inflammatory profile. It is possible that activated LXR at the monocyte level promotes its direct binding to regulatory regions of pro-inflammatory and/or epigenetic changes that favor the recruitment of other proinflammatory factors. Several mechanisms by activated LXRs might be altered that contribute to these transcriptional changes. 
LXR promotes a pro-inflammatory human macrophage signature

430

431

432

433

434 447 in other pathological settings where the presence of macrophages with deregulated anti448 inflammatory and immunosuppressive functions contribute to pathology (e.g., cancer).

osteoclast progenitors, where LXR agonists increased Mafb expression through Srebp-1c transactivation of the Mafb promoter, and no evidence was found for direct LXR binding to the Mafb promoter (73). Therefore, and regardless of the opposite effect of LXR on MAFB in human macrophages and mouse osteoclast progenitors, which might reflect species- or cell-specific differences (74), it appears that LXR is capable of pre-programming myeloid cells by altering the expression of critical regulators of differentiation and polarization.

In summary, we have described a critical role of the LXR nuclear receptors during monocyte-tomacrophage differentiation and in the acquisition of the transcriptional and functional profile of human monocyte-derived macrophages. LXR activation skews macrophages to acquire a more proinflammatory and inmuno-stimulatory phenotype. Thus, and considering the enhanced activity of LXR in animal models and human RA $(34,35,38)$, modulation of LXR appears as a potential therapeutic target, and LXR-dependent macrophage genes might be considered as useful prognostic/therapeutic markers for human inflammatory diseases. In this regard, it is worth mentioning that, when compared to TAM, RA synovial macrophages display the highest levels of MMP12, INHBA and CCL17 (24), whose expression we have now shown to be dependent on LXR. Therefore, it is tempting to speculate that enhancing the level of LXR activation might be beneficial 
LXR promotes a pro-inflammatory human macrophage signature

450

451

452

453

454

455

456

457

458

459

460

461

462

463

464

465

466

467

468

469

470

471

472

473

\section{FIGURE LEGENDS}

Figure 1. Differential expression of LXR and LXR-regulated genes in GM-M $\varnothing$ and M-M $\varnothing$. A. Schematic representation of the in vitro generation of pro-inflammatory (GM-M $\varnothing$ ) and antiinflammatory (M-M $\varnothing$ ) macrophages from peripheral blood human monocytes. B. GSEA of the gene sets upregulated or downregulated in rheumatoid arthritis synovial macrophages (52) (GSE10500, left panels) or preferentially expressed by large and small TAM (39)(right panels) on the ranked comparison of the M-M $\varnothing$ and GM-M $\varnothing$ transcriptomes. Normalized Enrichment Score (NES) and False Discovery rate q value (FDRq) are indicated in each case. C. Protein levels of LXR $\alpha$ and LXR $\beta$ in four independent samples (1-4) of GM-M $\varnothing$ and $M-M \varnothing$, as determined by Western blot, and using Vinculin and GAPDH as protein loading controls. D. LXR $\alpha$ and LXR $\beta$ expression in GM-M $\varnothing$ and M$M \emptyset$. Mean and SEM of 16 independent samples are shown. E. NR1H3 and NR1H2 mRNA expression in GM-M $\varnothing$ and M-M $\varnothing$. Mean and SEM of 20 independent samples are shown. F. Heatmap of the expression of desmosterol-upregulated genes in GM-M $\varnothing$ and M-M $\varnothing$. G. Relative mRNA expression of the indicated LXR target genes in GM-M $\varnothing$ and M-M $\varnothing$. Mean \pm SEM of six independent samples are shown. Statistical differences between groups were evaluated using paired t-test $\left(*, p<0.05 ;{ }^{* *}\right.$, $\mathrm{p}<0.01 ; * * *, \mathrm{p}<0.001 ; * * * *, \mathrm{p}<0.0001)$

Figure 2. Identification of LXR-regulated genes in anti-inflammatory M-M $\emptyset$. A. In vitro generation of control M-M $\varnothing$, GW3965-M-M $\varnothing$, GSK2033-M-M $\varnothing$ and GSK2033+GW3965-M-M $\varnothing$ before RNA isolation and RNA-sequencing. Control M-M $\varnothing$ were exposed to DMSO in parallel. B. GSEA of desmosterol-upregulated genes on the ranked comparison GW3965-M-M $\varnothing$ vs. M-M $\varnothing$ and GSK2033-M-M $\varnothing$ vs. M-M $\varnothing$. C. Number of differentially expressed genes $\left(\left|\log _{2} F C\right|>1, \operatorname{adjp}<0.05,\right)$ relative to control M-M $\varnothing$. D. Summary of GSEA of the "Pro-inflammatory gene set" and "Antiinflammatory gene set" on the ranked comparisons of the indicated transcriptomes. The color of 
LXR promotes a pro-inflammatory human macrophage signature

474 the circles illustrates the type of enrichment of each comparison (positive, red; negative, blue). The 475 area of each circle is proportional to the Normalized Enrichment Scores of each comparison, which 476 is also indicated. Two representative GSEA profiles are shown at the top. E. Unsupervised clustering 477 of differentially expressed genes $\left(\left|\log _{2} \mathrm{FC}\right|>1\right)$ between control M-M $\varnothing$ and the transcriptomes of M$478 M \emptyset$ generated in the presence of GW3965, GSK2033 or both. For each gene, mRNA expression level 479 in the three donors are represented after normalizing gene expression and k-means clustering using 480 Genesis (http://genome.tugraz.at/genesisclient/). F. Identification of "Pro-inflammatory gene set", 481 "Anti-inflammatory gene set" and desmosterol-upregulated genes in each cluster. G. Comparison of 482 the "Pro-inflammatory gene set" and "Anti-inflammatory gene set" with the genes significantly 483 regulated after M-M $\varnothing$ differentiation in the presence of GW3965 (upper panel) or GSK2033 (lower 484 panel).

485

Figure 3.- LXR activation in the generation of anti-inflammatory M-M $\varnothing$ : time-dependency and 487 functional consequences. A-B. Relative mRNA expression of the indicated genes from the "Anti488 inflammatory" (A) and "Pro-inflammatory" (B) gene sets in M-M $\varnothing$ generated under the indicated 489 treatments. $A B C A 1$ expression was evaluated as a readout for $L X R$ activation $(*, p<0.05 ; * *, p<0.01$; $490 * * *, p<0.001)$. C. Production of the indicated cytokines in $M-M \varnothing$ generated in the indicated 491 conditions, as determined by ELISA. Mean \pm SEM of six independent donors are shown $(*, p<0.05$; $492 * *, p<0.01)$. D. LPS-stimulated $(10 \mathrm{ng} / \mathrm{ml}, 18 \mathrm{~h})$ production of TNF, IFN $\beta 1$ and IL-1 $\beta$ by $\mathrm{M}-\mathrm{M} \varnothing$ 493 generated in the indicated conditions, as determined by ELISA. Mean \pm SEM of six independent 494 donors are shown $\left(*, p<0.05 ;{ }^{* *}, \mathrm{p}<0.01\right)$. E. T cell-stimulatory capacity of $\mathrm{M}-\mathrm{M} \varnothing$ generated in the 495 presence of GW3965 (1 $\mu \mathrm{M})$, or GSK2033 (1 $\mu \mathrm{M})$, as determined in Mixed Leukocyte Reactions (M$496 M \emptyset$ vs. allogeneic CD3+ T lymphocyte), and using M-M $\varnothing$ generated in the presence of DMSO as a 
497

498

499

500

501

502

503

504

505

506

507

508

509

510

511

512

513

514

515

control $(C N T M-M \emptyset)$. Mean \pm SEM of six independent experiments are shown. The relative T cellstimulatory capacity (\% Proliferation rate) is shown in the right panel $(*, p<0.05 ; * * * *, p<0.0001)$.

Figure 4. Identification of a novel set of LXR-dependent genes in proinflammatory GM-M $\emptyset$. A. In vitro generation of control GM-M $\varnothing$, GW3965-GM-M $\varnothing$, GSK2033-GM-M $\varnothing$ and GSK2033+GW3965GM-M $\varnothing$ before RNA isolation and RNA-sequencing. Control GM-M $\varnothing$ were exposed to DMSO in parallel. B. GSEA of desmosterol-upregulated genes in the ranked comparison GW3965-GM-M $\varnothing$ vs. GM-M $\varnothing$ and GSK2033-GM-M $\varnothing$ vs. GM-M $\varnothing$. C. Number of differentially expressed genes $\left(\left|\log _{2} \mathrm{FC}\right|>1\right.$, adjp $\left.<0.05,\right)$ relative to control GM-MØ. D. Summary of GSEA of the "Pro-inflammatory gene set" and "Anti-inflammatory gene set" on the ranked comparisons of the indicated transcriptomes. The color of the circles illustrates the type of enrichment of each comparison (positive, red; negative, blue). The area of each circle is proportional to the Normalized Enrichment Scores of each comparison, which is also indicated. Two representative GSEA profiles are shown at the top. E. Unsupervised clustering of differentially expressed genes $\left(\left|\log _{2} \mathrm{FC}\right|>1\right)$ between control GM-M $\varnothing$ and the transcriptomes of GM-M $\varnothing$ generated in the presence of GW3965, GSK2033 or both. For each gene, mRNA expression level in the three donors are represented after normalizing gene expression and k-means clustering using Genesis (http://genome.tugraz.at/genesisclient/). F. Indication of "Pro-inflammatory gene set", "Anti-inflammatory gene set" and desmosterolupregulated genes in each cluster. G. Comparison of the "Pro-inflammatory gene set" and "Antiinflammatory gene set" with the genes significantly regulated after GM-M $\varnothing$ differentiation in the presence of GW3965 (upper panel) or GSK2033 (lower panel).

Figure 5.- LXR activation in the generation of pro-inflammatory GM-M $\varnothing$ : time-dependency and functional consequences. A-B. Relative mRNA expression of the indicated genes from the "Pro- 
521

inflammatory" (A) and "Anti-inflammatory" (B) gene sets in GM-M $\varnothing$ generated under the indicated treatments. ABCA1 expression was evaluated as a readout for $\operatorname{LXR}$ activation $(*, \mathrm{p}<0.05 ; * *, \mathrm{p}<0.01$; $* * *, \mathrm{p}<0.001)$. C. Production of CCL17, IFN $\beta 1$ and IL-1 $\beta$ in GM-M $\varnothing$ generated in the indicated conditions, as determined by ELISA. Mean \pm SEM of seven independent donors are shown $(*, p<0.05$; ** $\mathrm{p}<0.01)$. D. LPS-induced (10 ng/ml, 18h) production of TNF and IL-10 in GM-M $\varnothing$ generated in the indicated conditions, as determined by ELISA. Mean \pm SEM of seven independent donors are shown $\left(*, p<0.05 ;{ }^{* *}, \mathrm{p}<0.01\right)$. The TNF/IL-10 is shown in the right panel. E. LPS-induced $(10 \mathrm{ng} / \mathrm{ml}, 18 \mathrm{~h})$ production of the indicated cytokines and chemokines in GM-M $\varnothing$ generated in the indicated conditions, as determined by ELISA. Mean \pm SEM of seven independent donors are shown $(*, p<0.05$; $\left.*^{*}, \mathrm{p}<0.01 ; * * *, \mathrm{p}<0.001\right)$. F-G. T cell-stimulatory capacity of GM-M $\varnothing$ generated in the presence of

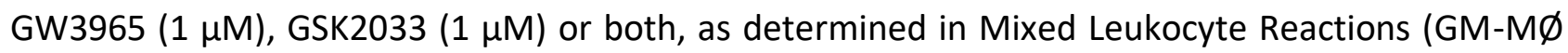
vs. allogeneic CD3+ T lymphocyte), and using GM-M $\varnothing$ generated in the presence of DMSO as a control (CNT GM-M $\varnothing$ ). After a 6-day coculture, ${ }^{3} \mathrm{H}$-thymidine incorporation was determined as a readout for $\mathrm{T}$ cell proliferation. Mean \pm SEM of six independent experiments are shown. The relative T cell-stimulatory capacity (\% Proliferation rate) is shown in $\mathbf{G}\left({ }^{*}, \mathrm{p}<0.05 ;{ }^{* *}, \mathrm{p}<0.01 ; * * *, \mathrm{p}<0.001\right.$; ****, $\mathrm{p}<0.0001)$. H. CD80 mRNA expression level in GM-M $\varnothing$ generated in the presence of GW3965 $(1 \mu \mathrm{M})$, GSK2033 $(1 \mu \mathrm{M})$ or both, as measured by the Expectation Maximization RNAseq (RSEM) algorithm, and using GM-M $\varnothing$ generated in the presence of DMSO as a control (CNT GM-M $\varnothing$ ). Adjusted $p$ values of indicated comparisons are shown.

\section{Figure 6. Molecular mechanisms underlying the macrophage polarizing effect of LXR. A. GSEA of} genes downregulated by siRNA MAFB in the ranked comparison of the transcriptomes of GSK2033GM-M $\varnothing$ and GM-M $\varnothing$. B. Relative protein levels of MAFB or MAF in M-M $\varnothing$ (left) and GM-M $\varnothing$ (right) generated in the presence of GW3965 (1 $\mu \mathrm{M})$, GSK2033 (1 $\mu \mathrm{M})$ or both, as determined by Western 
545 blot and densitometry, and using GM-M $\varnothing$ generated in the presence of DMSO as a control (insert).

546 For protein loading control purpose, Vinculin protein levels for determined in parallel. Mean \pm SEM 547 of the relative MAF and MAFB protein levels in M-M $\varnothing$ and GM-M $\varnothing$ from four independent donors 548 are shown, and a representative Western blot experiment is shown in each case. C. Production of 549 activin $A$ in $M-M \varnothing$ and GM-M $\varnothing$ generated in the presence of the LXR agonist GW3965 (1 $\mu M)$, LXR 550 inverse agonist GSK2033 $(1 \mu \mathrm{M})$ or both, as determined by ELISA, and using GM-M $\varnothing$ generated in 551 the presence of DMSO as a control. Mean \pm SEM of seven (GM-M $\varnothing)$ or eight (M-M $\varnothing)$ independent 552 donors are shown $(*, p<0.05 ; * *, p<0.01)$. D. Schematic representation of the assay to determine 553 the effect of the LXR inverse agonist GSK2033 on the expression of MAFB and the MAFB target gene 554 LGMN. E. Relative mRNA expression of the indicated genes in differentiating GM-M $\varnothing$ generated in 555 the presence of GSK2033 and after siRNA-mediated knock-down of MAFB. Mean \pm SEM of seven 556 independent experiments are shown $(*, p<0.05 ; * * p<0.01)$.

Figure 7. Physio-pathological relevance of the LXR-dependent expression of GM-M $\varnothing$-specific genes. A. Schematic representation for the generation of monocyte-derived macrophages in the 560 presence of ascitic fluid from tumor patients (AF-M $\varnothing$ ). B. Relative mRNA expression of the indicated 561 genes in M-M $\varnothing$ and AF-M $\varnothing$ generated in the absence of presence of GW3965. The results obtained 562 with each individual ascitic fluid are shown. C. Schematic representation for the generation of 563 monocyte-derived macrophages in the presence of Rheumatoid Arthritis synovial fluid (RASF-M $\varnothing$ ). 564 D. Expression of the indicated genes in RASF-M $\varnothing$ generated in the presence or absence of GSK2033. 565 Fold mRNA Expression indicates the expression of each gene relative to its expression in untreated 566 monocytes kept in culture for 72 hours. The results obtained with each individual synovial fluid are 567 shown. 
LXR promotes a pro-inflammatory human macrophage signature

568

569

570

571

572

573

\section{SUPPLEMENTARY FIGURE LEGENDS}

Supplementary Figure 1. A. Unsupervised clustering (http://genome.tugraz.at/genesisclient/) of differentially expressed genes $\left(\left|\log _{2} \mathrm{FC}\right|>1\right.$ and adjp<0.05) between control GM-M $\varnothing$ and the transcriptomes of GM-M $\varnothing$ generated in the presence of GW3965, GSK2033 or both. GSEA of the indicated gene clusters on the ranked list of the $M-M \varnothing$ and $G M-M \varnothing$ transcriptomes, and Gene ontology analysis of the gene clusters, as determined by statistical overrepresentation on the PANTHER (Protein ANalysis THrough Evolutionary Relationships) Classification System (http://www.pantherdb.org/about.jsp). Fold Enrichment and p-value of the gene ontology terms with a $p$ value $<0.05$ are shown. B. Normalized Enrichment Score (NES) of the transcriptomes of GM-M $\varnothing$ generated in the indicated conditions (insert) after GSEA using the Hallmarks gene sets from the Molecular Signatures Database v7.0 (http://software.broadinstitute.org/gsea/index.jsp) (FDR, False Discovery Rate).

Supplementary Figure 2. Distribution of Flag-LXR $\alpha$ tag densities in the vicinity of the indicated genes (highlighted in yellow) in colorectal adenocarcinoma HT29 cells treated with GW3965 (50). Data were obtained from the Cistrome Data Browser (GEO GSM2042848). The LXR $\alpha$ binding regions of interest (highlighted in orange) were scanned for LXRE motif using the HOMER motif matrix with DMINDA (https://bmbl.bmi.osumc.edu/DMINDA2/index.php) (51), and the corresponding statistical significance is shown.

Supplementary Figure 3. A. Expression of $L X R \alpha$ and $L X R \beta$ in terminally differentiated GM-M $\varnothing$ exposed to the LXR agonist GW3965 (1 $\mu \mathrm{M})$ or the LXR inverse agonist GSK2033 (1 $\mu \mathrm{M})$ for $24 \mathrm{~h}$ or 48h, as determined by Western blot, and using GM-M $\varnothing$ treated with DMSO for $48 \mathrm{~h}(-)$ as a control. For protein loading control purpose, vinculin and GAPDH protein levels for determined in parallel. 
B. Relative mRNA (NR1H2, NR1H3) and protein ( $L X R \alpha, L X R \beta)$ levels in terminally differentiated GMM $\varnothing$ exposed to GW3965 (1 $\mu \mathrm{M})$ or GSK2033 (1 $\mu \mathrm{M})$ for $24 \mathrm{~h}$ or $48 \mathrm{~h}$, as determined by qRT-PCR and 594 Western blot and densitometry, and using GM-M $\varnothing$ treated with DMSO for $48 \mathrm{~h}(-)$ as a control. Mean $595 \pm$ SEM of the relative levels of $N R 1 H 2, N R 1 H 3, L X R \alpha$ and LXR $\beta$ in GM-M $\varnothing$ from 16 (mRNA) or 20 596 (protein) independent donors are shown ( $\left.{ }^{*} p<0.05,{ }^{* *} p<0.01\right)$. C. Relative mRNA expression of the 597 indicated GM-M $\varnothing$ specific genes in terminally differentiated GM-M $\varnothing$ exposed to GW3965 (1 $\mu \mathrm{M})$ or 598 GSK2033 $(1 \mu \mathrm{M})$ for $24 \mathrm{~h}$ or $48 \mathrm{~h}$, as determined by qRT-PCR. Shown is the expression level of each 599 gene relative to its expression in vehicle (DMSO)-treated GM-M $\varnothing$. SREBF1, MERTK and ABCA1 gene 600 expression levels were determined as readouts for LXR activation. Mean \pm SEM of four independent $601 \mathrm{GM}-\mathrm{M} \varnothing$ samples are shown (* $\left.p<0.05,{ }^{* *} p<0.01\right)$. D. Schematic representation of the experiment 602 shown in panel B. E. Relative expression of the indicated genes from the "Pro-Inflammatory gene 603 set" and "Anti-Inflammatory gene set" in GM-M $\varnothing$ generated under low concentrations of GM-CSF 604 (one dose of $1000 \mathrm{U} / \mathrm{ml}$, or three doses of $50 \mathrm{U} / \mathrm{ml}$ ), and exposed to GW3965 (1 $\mu \mathrm{M})$, GSK2033 (1 $605 \mu \mathrm{M}$ ) or both, at day 5 of the differentiation process, and using GM-M $\varnothing$ generated in the presence 606 of DMSO as a control (insert). Mean \pm SEM of three independent experiments are shown ( ${ }^{*} p<0.05$, $607 * * p<0.01)$. 
LXR promotes a pro-inflammatory human macrophage signature

609

610

611

612

613

614

615

616

617

618

619

620

621

622

623

624

625

626

627

628

629

630

631

632

\section{DECLARATIONS}

Ethics approval and consent to participate. Biological samples were obtained from the Oncology Department (Hospital General Universitario Gregorio Marañón) after patients had provided informed consent. Approval was obtained from the ethics committee of Hospital General Universitario Gregorio Marañón. The procedures used in this study adhere to the tenets of the Declaration of Helsinki.

\section{Consent for publication. Not applicable}

Acknowledgements. This work was supported by grants from Ministerio de Ciencia, Investigación y Universidades (SAF2017-83785-R to MAV and ALC), Ministerio de Ciencia, Investigación y Universidades y Fondo Europeo de Desarrollo Regional (FEDER) (SAF2017-90604-REDT and PID2019104284RB-I00/AEI/10.13039/501100011033 to AC), Fundación La Marató/TV3 (Grant 201619.31 to ALC), Instituto de Salud Carlos III (Grant PI17/00037 to APK), and Red de Investigación en Enfermedades Reumáticas (RIER, RD16/0012/0007) from Instituto de Salud Carlos III and cofinanced by the European Regional Development Fund "A way to achieve Europe" (ERDF) to APK and ALC. AGA was funded by FPU predoctoral fellowship (FPU16/02756) from Ministerio de Ciencia e Innovación.

\section{Authors' information (optional)}

CONFLICTS OF INTEREST. The authors declare no competing financial interests.

AVAILABILITY OF DATA AND MATERIAL. All transcriptional information described in the manuscript has been deposited in Gene Expression Omnibus repository (http://www.ncbi.nlm.nih.gov/geo/) under accession GSE156783 and GSE156696.

AUTHORSHIP CONTRIBUTIONS. AGA, MT-T, JVR, BA and EC-S performed research and analyzed data; AGA, APK, MAV, AC and ALC designed the research and analyzed data; AGA, AC and ALC wrote the paper. 


\section{REFERENCES}

1. Hoeffel, G., and F. Ginhoux. 2018. Fetal monocytes and the origins of tissue-resident macrophages. Cell. Immunol. 330: 5-15.

2. Ruffell, B., and L. M. Coussens. 2015. Macrophages and therapeutic resistance in cancer. Cancer Cell 27: 462-472.

3. Lavin, Y., A. Mortha, A. Rahman, and M. Merad. 2015. Regulation of macrophage development and function in peripheral tissues. Nat Rev Immunol 15: 731-744.

4. Caescu, C. I., X. Guo, L. Tesfa, T. D. Bhagat, A. Verma, D. Zheng, and E. R. Stanley. 2015. Colony stimulating factor-1 receptor signaling networks inhibit mouse macrophage inflammatory responses by induction of microRNA-21. Blood 125: e1-e13.

5. Van Overmeire, E., B. Stijlemans, F. Heymann, J. Keirsse, Y. Morias, Y. Elkrim, L. Brys, C. Abels, Q. Lahmar, C. Ergen, L. Vereecke, F. Tacke, P. De Baetselier, J. A. Van Ginderachter, and D. Laoui. 2016. M-CSF and GM-CSF Receptor Signaling Differentially Regulate Monocyte Maturation and Macrophage Polarization in the Tumor Microenvironment. Cancer Res 76: 35-42.

6. Hamilton, J. A. 2008. Colony-stimulating factors in inflammation and autoimmunity. Nat. Rev. Immunol. 8: 533-544.

7. Ushach, I., and A. Zlotnik. 2016. Biological role of granulocyte macrophage colonystimulating factor (GM-CSF) and macrophage colony-stimulating factor (M-CSF) on cells of the myeloid lineage. J. Leukoc. Biol. 100: 481-489.

8. Yamamoto, T., C. Kaizu, T. Kawasaki, G. Hasegawa, H. Umezu, R. Ohashi, J. Sakurada, S. Jiang, L. Shultz, and M. Naito. 2008. Macrophage colony-stimulating factor is indispensable for repopulation and differentiation of Kupffer cells but not for splenic red pulp macrophages in osteopetrotic (op/op) mice after macrophage depletion. Cell Tissue Res. 332: 245-256.

9. Wynn, T. A., A. Chawla, and J. W. Pollard. 2013. Macrophage biology in development, homeostasis and disease. Nature 496: 445-455.

10. Fleetwood, A. J., H. Dinh, A. D. Cook, P. J. Hertzog, and J. A. Hamilton. 2009. GM-CSF- and M-CSF-dependent macrophage phenotypes display differential dependence on Type I interferon signaling. J. Leukoc. Biol. 86: 411-421.

11. Nowicki, A., J. Szenajch, G. Ostrowska, A. Wojtowicz, K. Wojtowicz, A. A. Kruszewski, M. Maruszynski, S. L. Aukerman, and W. Wiktor-Jedrzejczak. 1996. Impaired tumor growth in colony-stimulating factor 1 (CSF-1)-deficient, macrophage-deficient op/op mouse: Evidence for a role of CSF-1-dependent macrophages in formation of tumor stroma. Int. J. Cancer 65: 112-119.

12. Verreck, F. A. W., T. De Boer, D. M. L. Langenberg, M. A. Hoeve, M. Kramer, E. Vaisberg, R. Kastelein, A. Kolk, R. De Waal-Malefyt, and T. H. M. Ottenhoff. 2004. Human IL-23producing type 1 macrophages promote but IL-10-producing type 2 macrophages subvert immunity to (myco)bacteria. Proc. Natl. Acad. Sci. U. S. A. 101: 4560-4565. 13. Sierra-Filardi, E., A. Puig-Kröger, F. J. Blanco, C. Nieto, R. Bragado, M. I. Palomero, C. Bernabéu, M. A. Vega, and A. L. Corbí. 2011. Activin A skews macrophage polarization by promoting a proinflammatory phenotype and inhibiting the acquisition of anti-inflammatory macrophage markers. Blood 117: 5092-5101. 14. Kinoshita, M., T. Uchida, A. Sato, M. Nakashima, H. Nakashima, S. Shono, Y. Habu, H. Miyazaki, S. Hiroi, and S. Seki. 2010. Characterization of two F4/80-positive Kupffer cell subsets by their function and phenotype in mice. J Hepatol 53: 903-910. 
680

681

682

683

684

685

686

687

688

689

690

691

692

693

694

695

696

697

698

699

700

701

702

703

704

705

706

707

708

709

710

711

712

713

714

715

716

717

718

719

720

721

722

723

724

725

726

15. Movita, D., K. Kreefft, P. Biesta, A. van Oudenaren, P. J. M. Leenen, H. L. A. Janssen, and A. Boonstra. 2012. Kupffer cells express a unique combination of phenotypic and functional characteristics compared with splenic and peritoneal macrophages. J. Leukoc. Biol. 92: 723733.

16. Pyonteck, S. M., L. Akkari, A. J. Schuhmacher, R. L. Bowman, L. Sevenich, D. F. Quail, O. C. Olson, M. L. Quick, J. T. Huse, V. Teijeiro, M. Setty, C. S. Leslie, Y. Oei, A. Pedraza, J. Zhang, C. W. Brennan, J. C. Sutton, E. C. Holland, D. Daniel, and J. A. Joyce. 2013. CSF-1R inhibition alters macrophage polarization and blocks glioma progression. Nat. Med. 19: 1264-1272. 17. Pyonteck, S. M., B. B. Gadea, H. W. Wang, V. Gocheva, K. E. Hunter, L. H. Tang, and J. A. Joyce. 2012. Deficiency of the macrophage growth factor CSF-1 disrupts pancreatic neuroendocrine tumor development. Oncogene 31: 1459-1467.

18. Amemiya, H., H. Kono, and H. Fujii. 2011. Liver regeneration is impaired in macrophage colony stimulating factor deficient mice after partial hepatectomy: The role of M-CSFinduced macrophages. J. Surg. Res. 165: 59-67.

19. Kubota, Y., K. Takubo, T. Shimizu, H. Ohno, K. Kishi, M. Shibuya, H. Saya, and T. Suda. 2009. M-CSF inhibition selectively targets pathological angiogenesis and lymphangiogenesis. J. Exp. Med. 206: 1089-1102.

20. Fleetwood, A. J., T. Lawrence, J. A. Hamilton, and A. D. Cook. 2007. GranulocyteMacrophage Colony-Stimulating Factor (CSF) and Macrophage CSF-Dependent Macrophage Phenotypes Display Differences in Cytokine Profiles and Transcription Factor Activities: Implications for CSF Blockade in Inflammation. J. Immunol. 178: 5245-5252.

21. Lacey, D. C., A. Achuthan, A. J. Fleetwood, H. Dinh, J. Roiniotis, G. M. Scholz, M. W. Chang, S. K. Beckman, A. D. Cook, and J. A. Hamilton. 2012. Defining GM-CSF- and Macrophage-CSF-Dependent Macrophage Responses by In Vitro Models. J Immunol 188: 5752-5765.

22. Gonzalez-Dominguez, E., A. Dominguez-Soto, C. Nieto, J. L. Flores-Sevilla, M. PachecoBlanco, V. Campos-Pena, M. A. Meraz-Rios, M. A. Vega, A. L. Corbi, and C. Sanchez-Torres. 2016. Atypical Activin A and IL-10 Production Impairs Human CD16+ Monocyte Differentiation into Anti-Inflammatory Macrophages. J Immunol 196: 1327-1337. 23. Gonzalez-Dominguez, E., R. Samaniego, J. L. Flores-Sevilla, S. F. Campos-Campos, G. Gomez-Campos, A. Salas, V. Campos-Pena, A. L. Corbi, P. Sanchez-Mateos, and C. SanchezTorres. 2015. CD163L1 and CLEC5A discriminate subsets of human resident and inflammatory macrophages in vivo. J. Leukoc. Biol. 98: 453-466.

24. Palacios, B. S., L. Estrada-Capetillo, E. Izquierdo, G. Criado, C. Nieto, C. Municio, I. González-Alvaro, P. Sánchez-Mateos, J. L. Pablos, A. L. Corbí, and A. Puig-Kröger. 2015. Macrophages from the synovium of active rheumatoid arthritis exhibit an activin adependent pro-inflammatory profile. J. Pathol. 235: 515-526.

25. de las Casas-Engel, M., A. Dominguez-Soto, E. Sierra-Filardi, R. Bragado, C. Nieto, A. PuigKroger, R. Samaniego, M. Loza, M. T. Corcuera, F. Gomez-Aguado, M. Bustos, P. SanchezMateos, A. L. Corbi, M. Teresa Corcuera, F. Gomez-Aguado, M. Bustos, P. Sanchez-Mateos, and A. L. Corbi. 2013. Serotonin Skews Human Macrophage Polarization through HTR2B and HTR7. J Immunol 190: 2301-2310.

26. Municio, C., Á. Dominguez-Soto, S. Fuentelsaz-Romero, A. Lamana, N. Montes, V. D. Cuevas, R. G. Campos, J. L. Pablos, I. González-Álvaro, and A. Puig-Kröger. 2018. Methotrexate limits inflammation through an A20-dependent cross-tolerance mechanism. Ann. Rheum. Dis. 77: 752-759.

27. Municio, C., B. Soler Palacios, L. Estrada-Capetillo, A. Benguria, A. Dopazo, E. Garcia- 
Lorenzo, S. Fernandez-Arroyo, J. Joven, M. E. Miranda-Carus, I. Gonzalez-Alvaro, and A. PuigKroger. 2016. Methotrexate selectively targets human proinflammatory macrophages through a thymidylate synthase/p53 axis. Ann Rheum Dis 75: 2157-2165.

28. El-Hajjaji, F. Z., A. Oumeddour, A. J. C. Pommier, A. Ouvrier, E. Viennois, J. Dufour, F. Caira, J. R. Drevet, D. H. Volle, S. Baron, F. Saez, and J. M. A. Lobaccaro. 2011. Liver X receptors, lipids and their reproductive secrets in the male. Biochim. Biophys. Acta - Mol. Basis Dis. 1812: 974-981.

29. Schulman, I. G. 2017. Liver $X$ receptors link lipid metabolism and inflammation. FEBS Lett. 591: 2978-2991.

30. Kiss, M., Z. Czimmerer, and L. Nagy. 2013. The role of lipid-activated nuclear receptors in shaping macrophage and dendritic cell function: From physiology to pathology. J. Allergy Clin. Immunol. 132: 264-286.

31. Joseph, S. B., A. Castrillo, B. A. Laffitte, D. J. Mangelsdorf, and P. Tontonoz. 2003. Reciprocal regulation of inflammation and lipid metabolism by liver $\mathrm{X}$ receptors. Nat. Med. 9: 213-219.

32. Joseph, S. B., E. McKilligin, L. Pei, M. A. Watson, A. R. Collins, B. A. Laffitte, M. Chen, G. Noh, J. Goodman, G. N. Hagger, J. Tran, T. K. Tippin, X. Wang, A. J. Lusis, W. A. Hsueh, R. E. Law, J. L. Collins, T. M. Willson, and P. Tontonoz. 2002. Synthetic LXR ligand inhibits the development of atherosclerosis in mice. Proc. Natl. Acad. Sci. U. S. A. 99: 7604-7609. 33. Fontaine, C., E. Rigamonti, A. Nohara, P. Gervois, E. Teissier, J. C. Fruchart, B. Staels, and G. Chinetti-Gbaguidi. 2007. Liver $X$ receptor activation potentiates the lipopolysaccharide response in human macrophages. Circ. Res. 101: 40-49.

34. Asquith, D. L., A. M. Miller, A. J. Hueber, H. J. McKinnon, N. Sattar, G. J. Graham, and I. B. McInnes. 2009. Liver $X$ receptor agonism promotes articular inflammation in murine collagen-induced arthritis. Arthritis Rheum. 60: 2655-2665.

35. Asquith, D. L., A. M. Miller, J. Reilly, S. Kerr, P. Welsh, N. Sattar, and I. B. Mclnnes. 2011. Simultaneous activation of the liver $X$ receptors ( $L X R \alpha$ and $L X R \beta)$ drives murine collageninduced arthritis disease pathology. Ann. Rheum. Dis. 70: 2225-2228. 36. Töröcsik, D., M. Baráth, S. Benkő, L. Széles, B. Dezső, S. Póliska, Z. Hegyi, L. Homolya, I. Szatmári, Á. Lányi, and L. Nagy. 2010. Activation of Liver X Receptor Sensitizes Human Dendritic Cells to Inflammatory Stimuli. J. Immunol. 184: 5456-5465.

37. Tavazoie, M. F., I. Pollack, R. Tanqueco, B. N. Ostendorf, B. S. Reis, F. C. Gonsalves, I. Kurth, C. Andreu-Agullo, M. L. Derbyshire, J. Posada, S. Takeda, K. N. Tafreshian, E. Rowinsky, M. Szarek, R. J. Waltzman, E. A. Mcmillan, C. Zhao, M. Mita, A. Mita, B. Chmielowski, M. A. Postow, A. Ribas, D. Mucida, and S. F. Tavazoie. 2018. LXR/ApoE Activation Restricts Innate Immune Suppression in Cancer. Cell 172: 825-840.e18.

38. Asquith, D. L., L. E. Ballantine, J. S. Nijjar, M. K. Makdasy, S. Patel, P. B. Wright, J. H. Reilly, S. Kerr, M. Kurowska-Stolarska, J. A. Gracie, and I. B. McInnes. 2013. The liver X receptor pathway is highly upregulated in rheumatoid arthritis synovial Macrophages and potentiates TLR-driven cytokine release. Ann. Rheum. Dis. 72: 2024-2031.

39. Donadon, M., G. Torzilli, N. Cortese, C. Soldani, L. Di Tommaso, B. Franceschini, R. Carriero, M. Barbagallo, A. Rigamonti, A. Anselmo, F. S. Colombo, G. Maggi, A. Lleo, J. Cibella, C. Peano, P. Kunderfranco, M. Roncalli, A. Mantovani, and F. Marchesi. 2020. Macrophage morphology correlates with single-cell diversity and prognosis in colorectal liver metastasis. J. Exp. Med. 217.

40. Sica, A., and A. Mantovani. 2012. Macrophage plasticity and polarization: in vivo veritas. J Clin Invest 122: 787-795. 
41. Sohrabi, Y., G. V. H. Sonntag, L. C. Braun, S. M. M. Lagache, M. Liebmann, L. Klotz, R. Godfrey, F. Kahles, J. Waltenberger, and H. M. Findeisen. 2020. LXR Activation Induces a Proinflammatory Trained Innate Immunity-Phenotype in Human Monocytes. Front. Immunol. 11: 353.

42. Cuevas, V. D., L. Anta, R. Samaniego, E. Orta-Zavalza, J. Vladimir de la Rosa, G. Baujat, A. Dominguez-Soto, P. Sanchez-Mateos, M. M. Escribese, A. Castrillo, V. Cormier-Daire, M. A. Vega, and A. L. Corbi. 2017. MAFB Determines Human Macrophage Anti-Inflammatory Polarization: Relevance for the Pathogenic Mechanisms Operating in Multicentric Carpotarsal Osteolysis. J Immunol 198: 2070-2081.

43. Langmead, B., and S. L. Salzberg. 2012. Fast gapped-read alignment with Bowtie 2. Nat. Methods 9: 357-359.

44. Li, B., and C. N. Dewey. 2011. RSEM: Accurate transcript quantification from RNA-Seq data with or without a reference genome. BMC Bioinformatics 12. 45. Sturn, A., J. Quackenbush, and Z. Trajanoski. 2002. Genesis: cluster analysis of microarray data. Bioinformatics 18: 207-208.

46. Chen, E. Y., C. M. Tan, Y. Kou, Q. Duan, Z. Wang, G. V. Meirelles, N. R. Clark, and A. Ma'ayan. 2013. Enrichr: Interactive and collaborative HTML5 gene list enrichment analysis tool. BMC Bioinformatics 14: 128.

47. Kuleshov, M. V, M. R. Jones, A. D. Rouillard, N. F. Fernandez, Q. Duan, Z. Wang, S. Koplev, S. L. Jenkins, K. M. Jagodnik, A. Lachmann, M. G. McDermott, C. D. Monteiro, G. W. Gundersen, and A. Ma'ayan. 2016. Enrichr: a comprehensive gene set enrichment analysis web server 2016 update. Nucleic Acids Res 44: W90-W97.

48. Mi, H., A. Muruganujan, and P. D. Thomas. 2013. PANTHER in 2013: Modeling the evolution of gene function, and other gene attributes, in the context of phylogenetic trees. Nucleic Acids Res. 41.

49. Subramanian, A., P. Tamayo, V. K. Mootha, S. Mukherjee, B. L. Ebert, M. A. Gillette, A. Paulovich, S. L. Pomeroy, T. R. Golub, E. S. Lander, and J. P. Mesirov. 2005. Gene set enrichment analysis: a knowledge-based approach for interpreting genome-wide expression profiles. Proc Natl Acad Sci U S A 102: 15545-15550.

50. Savic, D., R. C. Ramaker, B. S. Roberts, E. C. Dean, T. C. Burwell, S. K. Meadows, S. J. Cooper, M. J. Garabedian, J. Gertz, and R. M. Myers. 2016. Distinct gene regulatory programs define the inhibitory effects of liver $\mathrm{X}$ receptors and PPARG on cancer cell proliferation. Genome Med. 8.

51. Ma, Q., H. Zhang, X. Mao, C. Zhou, B. Liu, X. Chen, and Y. Xu. 2014. DMINDA: An integrated web server for DNA motif identification and analyses. Nucleic Acids Res. 42. 52. Yarilina, A., K. H. Park-Min, T. Antoniv, X. Hu, and L. B. Ivashkiv. 2008. TNF activates an IRF1-dependent autocrine loop leading to sustained expression of chemokines and STAT1dependent type I interferon-response genes. Nat. Immunol. 9: 378-387.

53. Muse, E. D., S. Yu, C. R. Edillor, J. Tao, N. J. Spann, T. D. Troutman, J. S. Seidman, A. Henke, J. T. Roland, K. A. Ozeki, B. M. Thompson, J. G. McDonald, J. Bahadorani, S. Tsimikas, T. R. Grossman, M. S. Tremblay, and C. K. Glass. 2018. Cell-specific discrimination of desmosterol and desmosterol mimetics confers selective regulation of LXR and SREBP in macrophages. Proc. Natl. Acad. Sci. U. S. A. 115: E4680-E4689.

54. Zhang, Y., J. J. Repa, K. Gauthier, and D. J. Mangelsdorf. 2001. Regulation of Lipoprotein Lipase by the Oxysterol Receptors, LXR $\alpha$ and LXRß. J. Biol. Chem. 276: 43018-43024.

55. Beyea, M. M., C. L. Heslop, C. G. Sawyez, J. Y. Edwards, J. G. Markle, R. A. Hegele, and M. W. Huff. 2007. Selective up-regulation of LXR-regulated genes ABCA1, ABCG1, and APOE in 
macrophages through increased endogenous synthesis of 24(S),25-epoxycholesterol. J. Biol. Chem. 282: 5207-5216.

56. Costet, P., Y. Luo, N. Wang, and A. R. Tall. 2000. Sterol-dependent transactivation of the $A B C 1$ promoter by the liver $X$ receptor/retinoid X receptor. J. Biol. Chem. 275: 28240-28245. 57. Engel, T., S. Lorkowski, A. Lueken, S. Rust, B. Schlüter, G. Berger, P. Cullen, and G. Assmann. 2001. The human ABCG4 gene is regulated by oxysterols and retinoids in monocyte-derived macrophages. Biochem. Biophys. Res. Commun. 288: 483-488. 58. A-Gonzalez, N., S. J. Bensinger, C. Hong, S. Beceiro, M. N. Bradley, N. Zelcer, J. Deniz, C. Ramirez, M. Díaz, G. Gallardo, C. Ruiz de Galarreta, J. Salazar, F. Lopez, P. Edwards, J. Parks, M. Andujar, P. Tontonoz, and A. Castrillo. 2009. Apoptotic Cells Promote Their Own Clearance and Immune Tolerance through Activation of the Nuclear Receptor LXR. Immunity 31: 245-258.

59. Hong, C., R. Walczak, H. Dhamko, M. N. Bradley, C. Marathe, R. Boyadjian, J. V. Salazar, and P. Tontonoz. 2011. Constitutive activation of LXR in macrophages regulates metabolic and inflammatory gene expression: Identification of ARL7 as a direct target. J. Lipid Res. 52: 531-539.

60. Marathe, C., M. N. Bradley, C. Hong, F. Lopez, C. M. Ruiz De Galarreta, P. Tontonoz, and A. Castrillo. 2006. The arginase II gene is an anti-inflammatory target of liver $\mathrm{X}$ receptor in macrophages. J. Biol. Chem. 281: 32197-32206.

61. Mak, P. A., H. R. Kast-Woelbern, A. M. Anisfeld, and P. A. Edwards. 2002. Identification of PLTP as an LXR target gene and apoE as an FXR target gene reveals overlapping targets for the two nuclear receptors. J. Lipid Res. 43: 2037-2041.

62. Domínguez-Soto, A., E. Sierra-Filardi, A. Puig-Kröger, B. Pérez-Maceda, F. Gómez-Aguado, M. T. Corcuera, P. Sánchez-Mateos, and A. L. Corbí. 2011. Dendritic Cell-Specific ICAM-3Grabbing Nonintegrin Expression on M2-Polarized and Tumor-Associated Macrophages Is Macrophage-CSF Dependent and Enhanced by Tumor-Derived IL-6 and IL-10. J. Immunol. 186: 2192-2200.

63. Puig-Kröger, A., E. Sierra-Filardi, A. Domínguez-Soto, R. Samaniego, M. T. Corcuera, F. Gómez-Aguado, M. Ratnam, P. Sánchez-Mateos, and A. L. Corbí. 2009. Folate receptor $\beta$ is expressed by tumor-associated macrophages and constitutes a marker for M2 antiinflammatory/regulatory Macrophages. Cancer Res. 69: 9395-9403.

64. Kim, H. 2017. The transcription factor MafB promotes anti-inflammatory M2 polarization and cholesterol efflux in macrophages. Sci. Rep. 7.

65. Liu, M., Z. Tong, C. Ding, F. Luo, S. Wu, C. Wu, S. Albeituni, L. He, X. Hu, D. Tieri, E. C. Rouchka, M. Hamada, S. Takahashi, A. A. Gibb, G. Kloecker, H. G. Zhang, M. Bousamra, B. G. Hill, X. Zhang, and J. Yan. 2020. Transcription factor c-Maf is a checkpoint that programs macrophages in lung cancer. J. Clin. Invest. 130: 2081-2096.

66. Kasprzak, A., and W. Szaflarski. 2020. Role of alternatively spliced messenger RNA (mRNA) isoforms of the insulin-like growth factor 1 (IGF1) in selected human tumors. Int. J. Mol. Sci. 21: 1-33.

67. Christopoulos, P. F., A. Corthay, and M. Koutsilieris. 2018. Aiming for the Insulin-like Growth Factor-1 system in breast cancer therapeutics. Cancer Treat. Rev. 63: 79-95. 68. Samaniego, R., Á. Domínguez-Soto, M. Ratnam, T. Matsuyama, P. Sánchez-Mateos, Á. L. Corbí, and A. Puig-Kröger. 2020. Folate Receptor $\beta$ (FR $\beta$ ) Expression in Tissue-Resident and Tumor-Associated Macrophages Associates with and Depends on the Expression of PU.1. Cells 9. 69. A-González, N., and A. Castrillo. 2011. Liver X receptors as regulators of macrophage 
868 inflammatory and metabolic pathways. Biochim. Biophys. Acta - Mol. Basis Dis. 1812: 982869994.

870 70. N, A. G., J. A. Guillen, G. Gallardo, M. Diaz, J. V de la Rosa, I. H. Hernandez, M. Casanova871 Acebes, F. Lopez, C. Tabraue, S. Beceiro, C. Hong, P. C. Lara, M. Andujar, S. Arai, T. Miyazaki, 872 S. Li, A. L. Corbi, P. Tontonoz, A. Hidalgo, and A. Castrillo. 2013. The nuclear receptor 873 LXRalpha controls the functional specialization of splenic macrophages. Nat Immunol 14: 874 831-839.

875 71. Cao, S., J. Liu, L. Song, and X. Ma. 2005. The Protooncogene c-Maf Is an Essential 876 Transcription Factor for IL-10 Gene Expression in Macrophages. J. Immunol. 174: 3484-3492. 877 72. Kikuchi, K., M. Iida, N. Ikeda, S. Moriyama, M. Hamada, S. Takahashi, H. Kitamura, T. 878 Watanabe, Y. Hasegawa, K. Hase, T. Fukuhara, H. Sato, E. H. Kobayashi, T. Suzuki, M. 879 Yamamoto, M. Tanaka, and K. Asano. 2018. Macrophages Switch Their Phenotype by 880 Regulating Maf Expression during Different Phases of Inflammation. J. Immunol. 201: 635881651.

882 73. Menendez-Gutierrez, M. P., T. Roszer, L. Fuentes, V. Nunez, A. Escolano, J. M. Redondo, 883 N. De Clerck, D. Metzger, A. F. Valledor, and M. Ricote. 2015. Retinoid X receptors 884 orchestrate osteoclast differentiation and postnatal bone remodeling. J Clin Invest 125: 809885823.

886 74. Laffitte, B. A., S. B. Joseph, R. Walczak, L. Pei, D. C. Wilpitz, J. L. Collins, and P. Tontonoz. 887 2001. Autoregulation of the Human Liver X Receptor $\alpha$ Promoter. Mol. Cell. Biol. 21: 75588887568. 


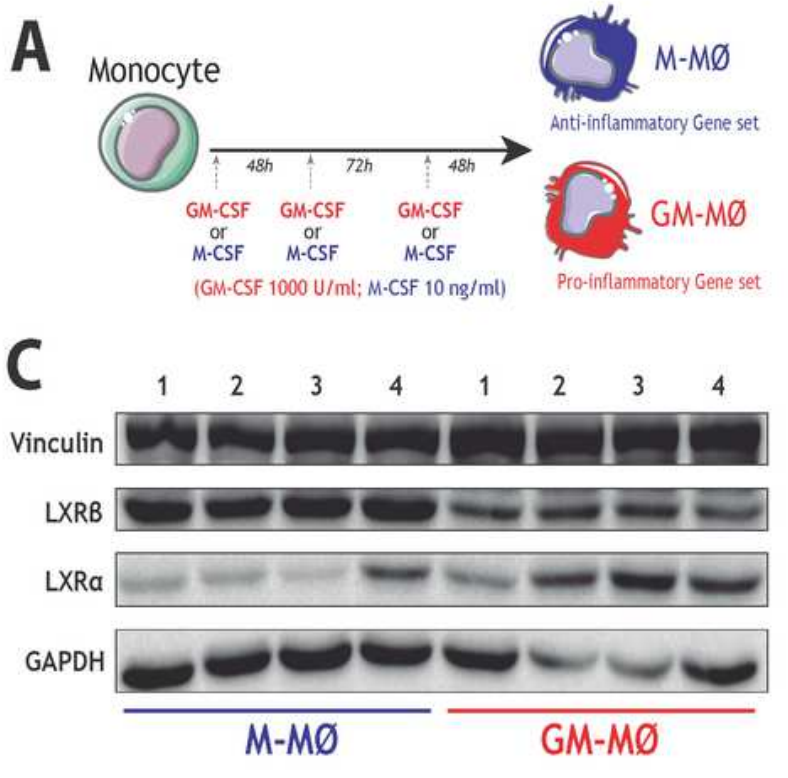

D

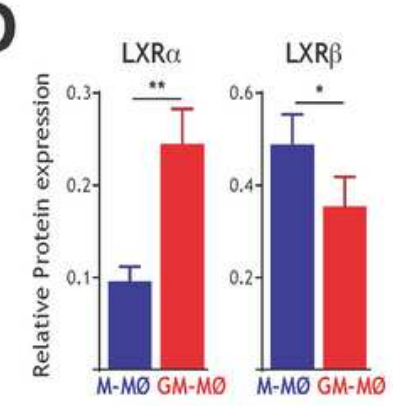

E

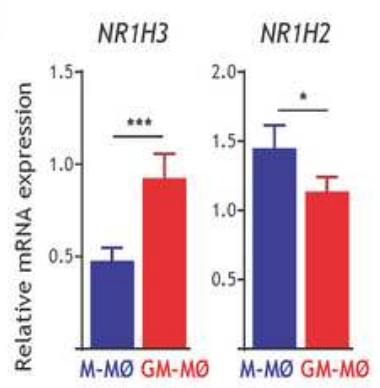

B

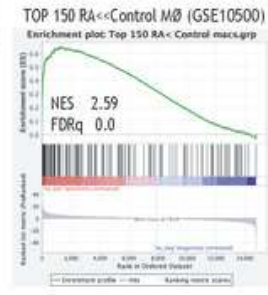

TOP 150 RA P > Control MD (GSE10500)

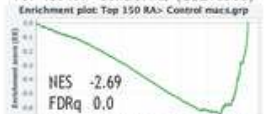

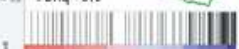
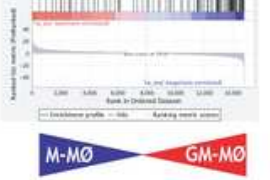

TOP 150 Large TAM geneset

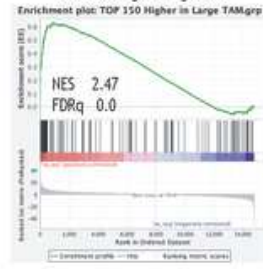

TOP 150 Small TA geneset

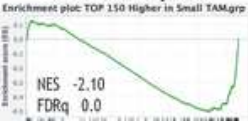

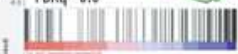

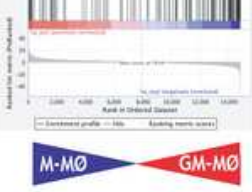

$\mathbf{F}$

$\mathbf{G}$
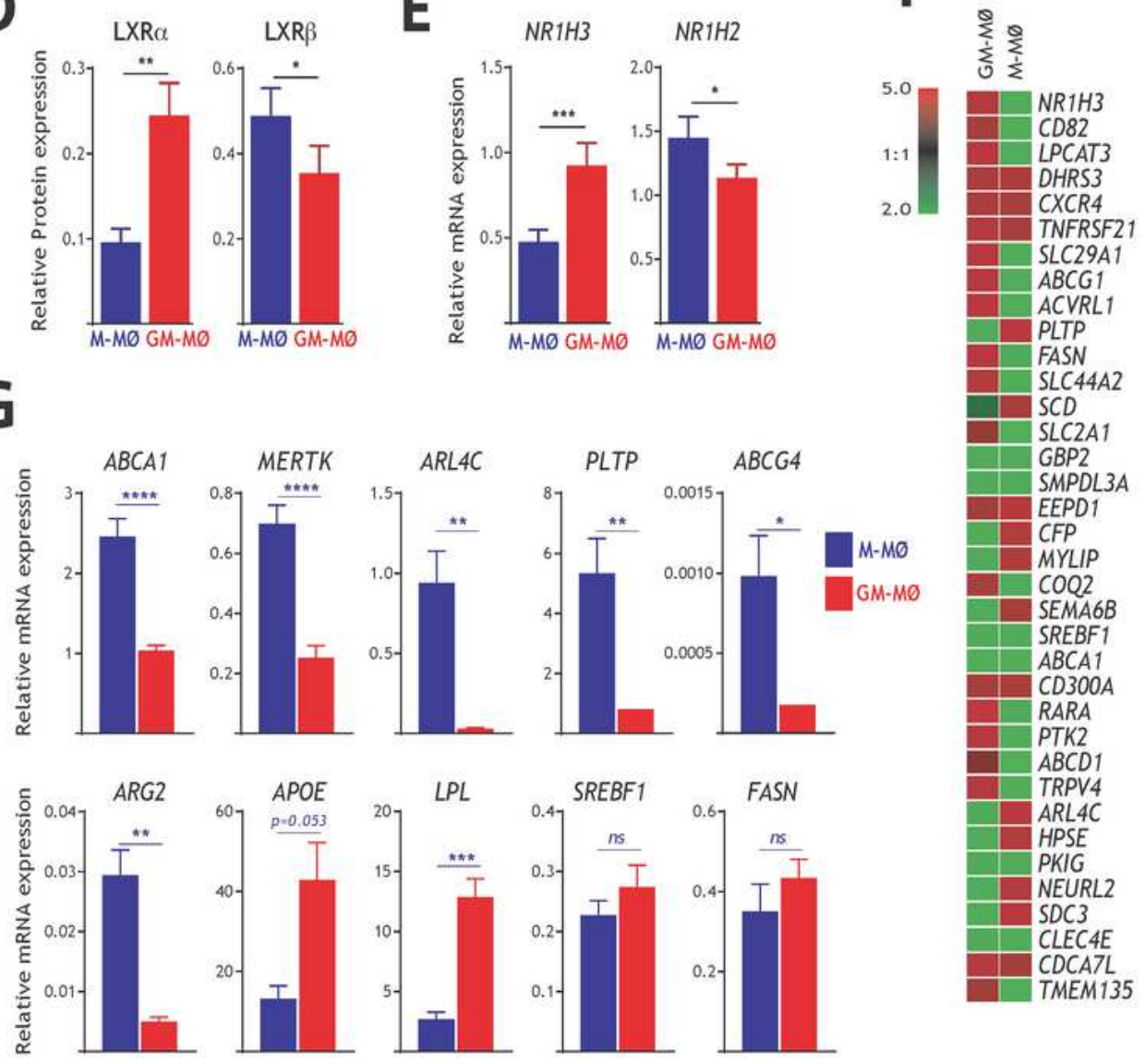

Figure 1

\section{Figure 1}

Differential expression of LXR and LXR-regulated genes in GM-M $\varnothing$ and M-M $\varnothing$. A. Schematic representation of the in vitro generation of pro-inflammatory (GM-M $\varnothing)$ and anti- inflammatory (M-M $\varnothing$ ) macrophages from peripheral blood human monocytes. B. GSEA of the gene sets upregulated or 
downregulated in rheumatoid arthritis synovial macrophages (52) (GSE10500, left panels) or preferentially expressed by large and small TAM (39)(right panels) on the ranked comparison of the M$\mathrm{M} \varnothing$ and GM-M $\varnothing$ transcriptomes. Normalized Enrichment Score (NES) and False Discovery rate q value (FDRq) are indicated in each case. C. Protein levels of $L X R a$ and $L X R \beta$ in four independent samples (1-4) of GM-M $\varnothing$ and M-M $\varnothing$, as determined by Western blot, and using Vinculin and GAPDH as protein loading controls. D. LXRa and LXR $\beta$ expression in GM-M $\varnothing$ and M- MØ. Mean and SEM of 16 independent samples are shown. E. NR1H3 and NR1H2 mRNA expression in GM-M $\varnothing$ and M-M $\emptyset$. Mean and SEM of 20 independent samples are shown. F. Heatmap of the expression of desmosterol-upregulated genes in GM$M \emptyset$ and M-M $\varnothing$. G. Relative mRNA expression of the indicated LXR target genes in GM-M $\varnothing$ and M-M $\varnothing$. Mean \pm SEM of six independent samples are shown. Statistical differences between groups were evaluated using paired t-test $(*, p<0.05 ; * \star, p<0.01 ; * \star *, p<0.001 ; * \star \star *, p<0.0001)$.
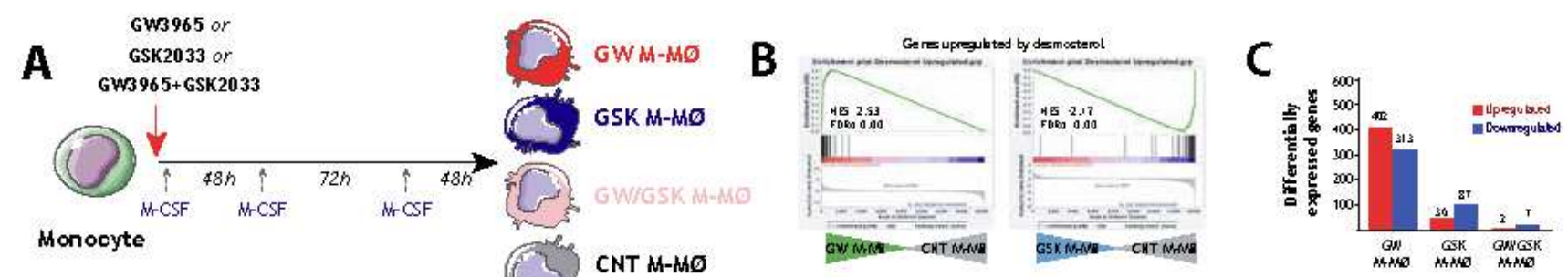

D

E

CNT M-Mø
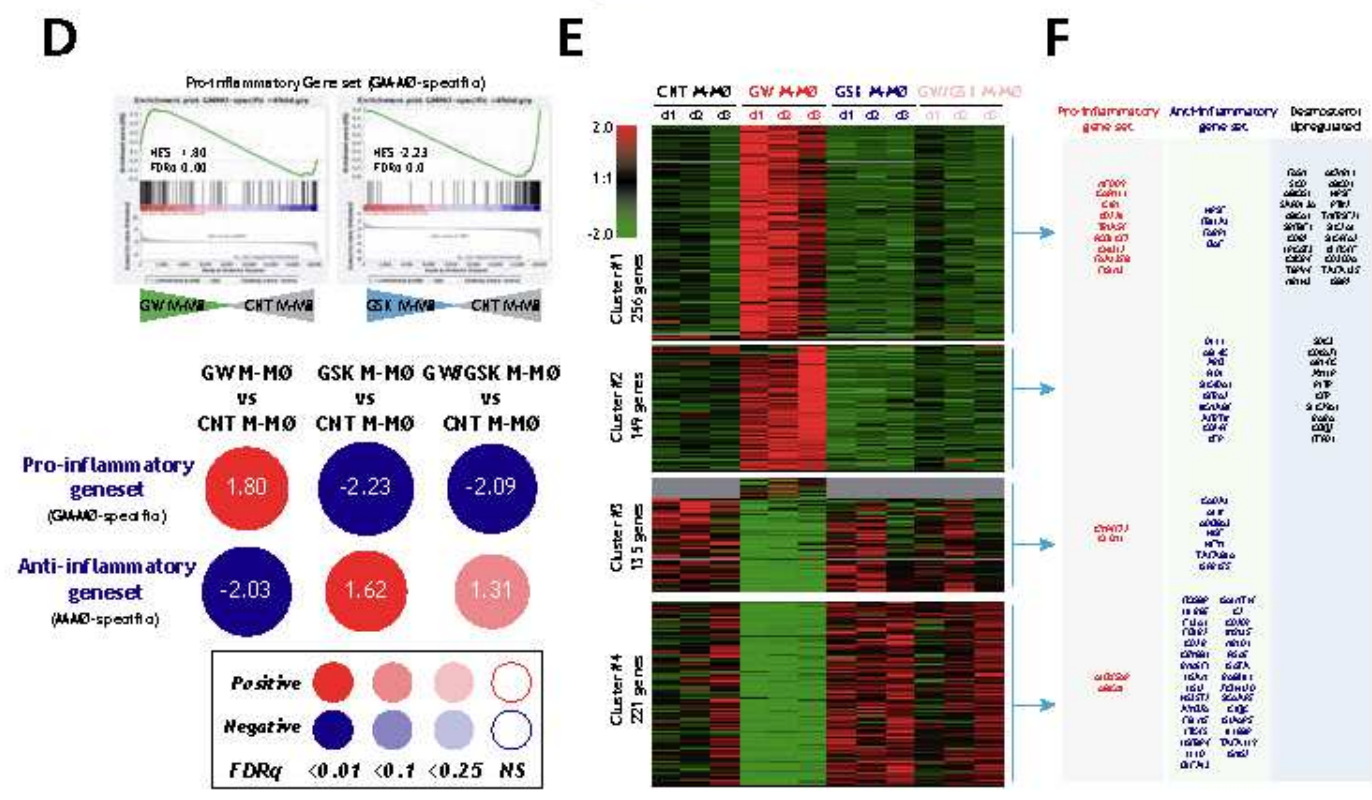

G

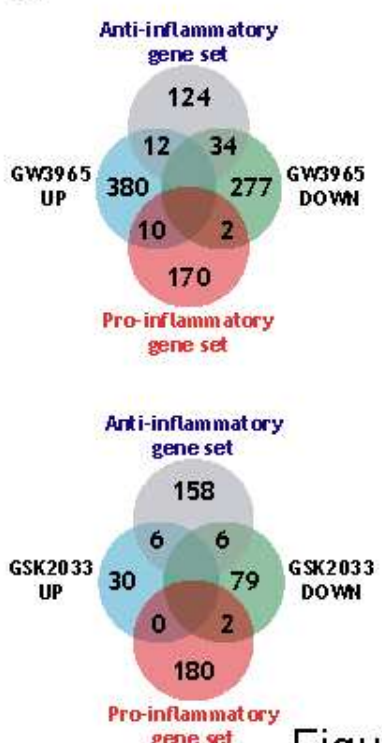

Figure 2

Figure 2

Identification of LXR-regulated genes in anti-inflammatory M-MØ. A. In vitro generation of control M-M $\varnothing$, GW3965-M-M Ø, GSK2033-M-MØ and GSK2033+GW3965-M-M Ø before RNA isolation and RNAsequencing. Control M-MØ were exposed to DMSO in parallel. B. GSEA of desmosterol-upregulated genes on the ranked comparison GW3965-M-M $\varnothing$ vs. M-M $\emptyset$ and GSK2033-M-M $\varnothing$ vs. M-MØ. C. Number of differentially expressed genes ( $|\log 2 \mathrm{FC}|>1$, adjp $<0.05$,) relative to control M-M $\emptyset$. D. Summary of GSEA of the "Pro-inflammatory gene set" and "Anti- inflammatory gene set" on the ranked comparisons of the indicated transcriptomes. The color of the circles illustrates the type of enrichment of each comparison 
(positive, red; negative, blue). The area of each circle is proportional to the Normalized Enrichment Scores of each comparison, which is also indicated. Two representative GSEA profiles are shown at the top. E. Unsupervised clustering of differentially expressed genes ( $|\log 2 \mathrm{FC}|>1)$ between control M-M $\varnothing$ and the transcriptomes of M- M $\varnothing$ generated in the presence of GW3965, GSK2033 or both. For each gene, mRNA expression level in the three donors are represented after normalizing gene expression and k-means clustering using Genesis (http://genome.tugraz.at/genesisclient/). F. Identification of "Pro-inflammatory gene set", "Anti-inflammatory gene set" and desmosterol-upregulated genes in each cluster. G.

Comparison of the "Pro-inflammatory gene set" and "Anti-inflammatory gene set" with the genes significantly regulated after M-M $\varnothing$ differentiation in the presence of GW3965 (upper panel) or GSK2033 (lower 483 panel).
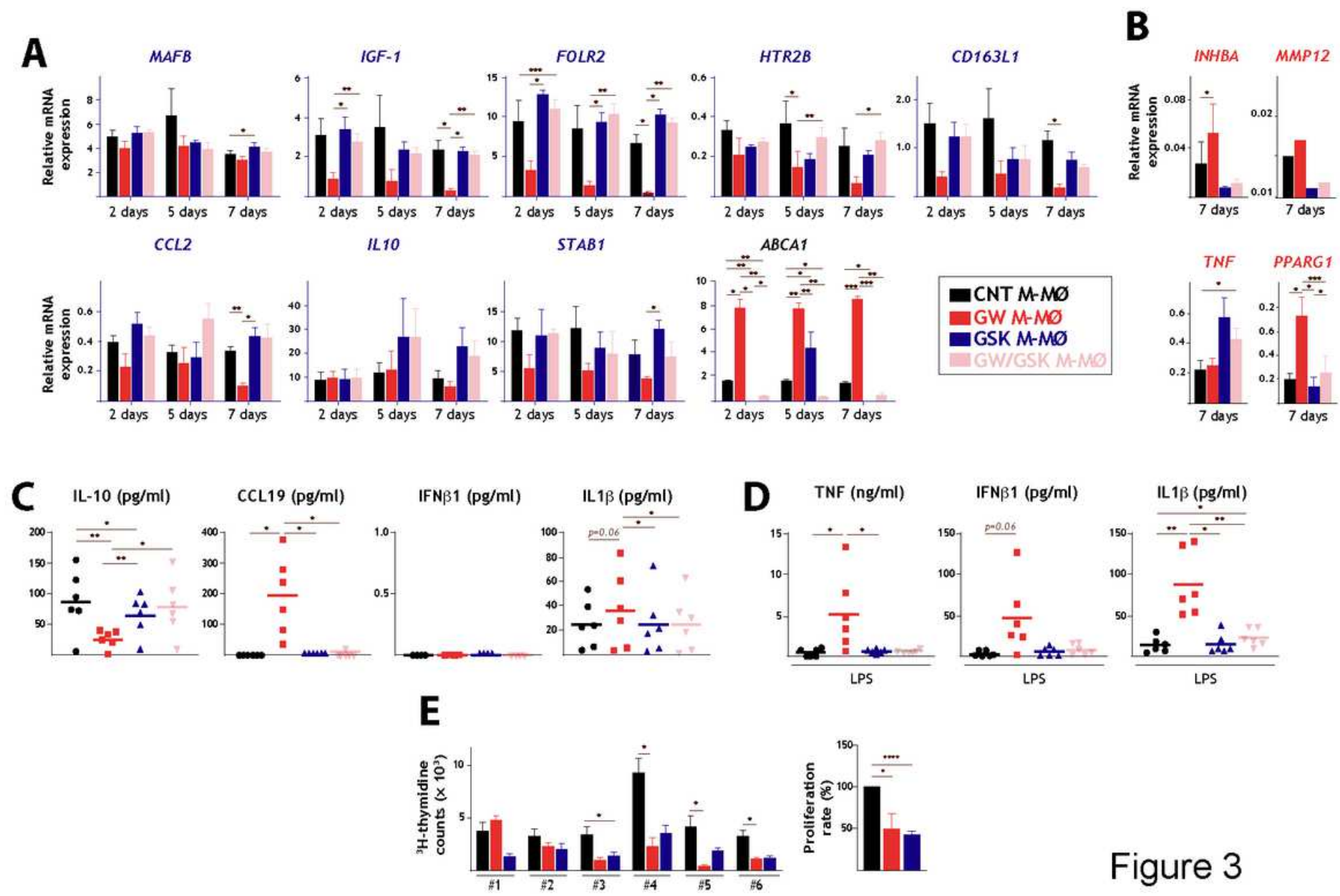

Figure 3

\section{Figure 3}

LXR activation in the generation of anti-inflammatory M-MØ: time-dependency and functional consequences. A-B. Relative mRNA expression of the indicated genes from the "Anti- inflammatory" (A) and "Pro-inflammatory" (B) gene sets in M-M $\emptyset$ generated under the indicated treatments. ABCA1 expression was evaluated as a readout for $\operatorname{LXR}$ activation ( $\left.{ }^{*}, \mathrm{p}<0.05 ; * \star, p<0.01 ; * \star *, p<0.001\right)$. C. Production of the indicated cytokines in M-M $\varnothing$ generated in the indicated conditions, as determined by ELISA. Mean \pm SEM of six independent donors are shown ( $*, p<0.05 ; * \star, p<0.01)$. D. LPS-stimulated $(10$ 


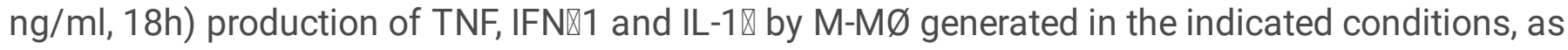
determined by ELISA. Mean \pm SEM of six independent donors are shown $\left({ }^{*}, p<0.05 ; * \star, p<0.01\right)$. E. T cellstimulatory capacity of M-MØ generated in the presence of GW3965 $(1 \mu \mathrm{M})$, or GSK2033 $(1 \mu \mathrm{M})$, as determined in Mixed Leukocyte Reactions (M- MØ vs. allogeneic CD3+ T lymphocyte), and using M-M $\varnothing$ generated in the presence of DMSO as a control (CNT M-M Ø). Mean \pm SEM of six independent experiments are shown. The relative T cell- stimulatory capacity (\% Proliferation rate) is shown in the right panel $(*, p<0.05 ; * \star \star \star, p<0.0001)$.

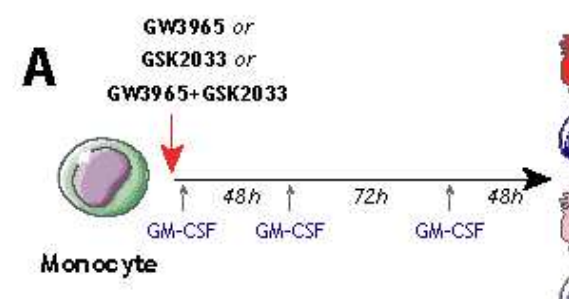

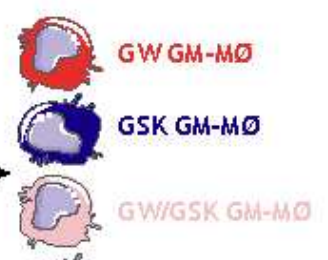

CNT GM-HO
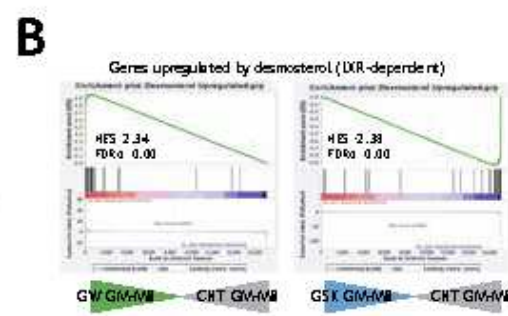

\section{$\mathrm{E}$}

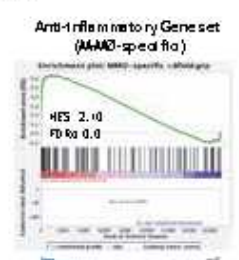

G5X GQros- CHT GWHU
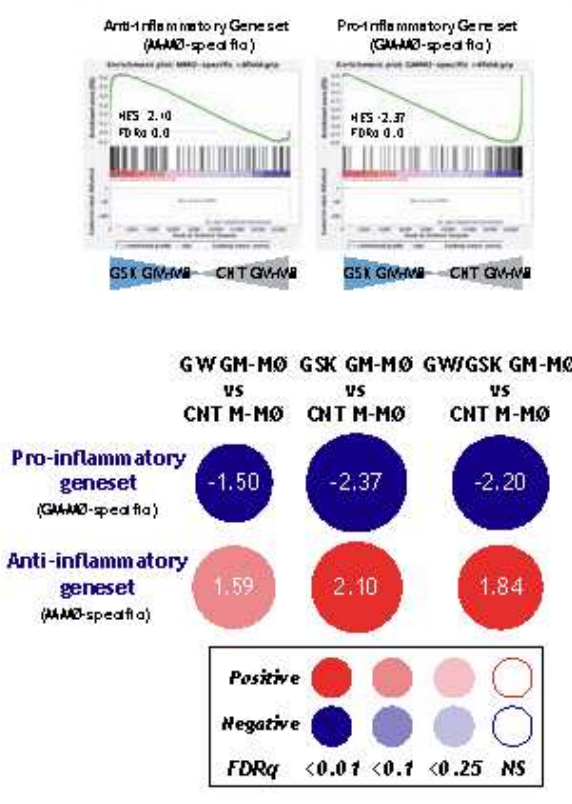
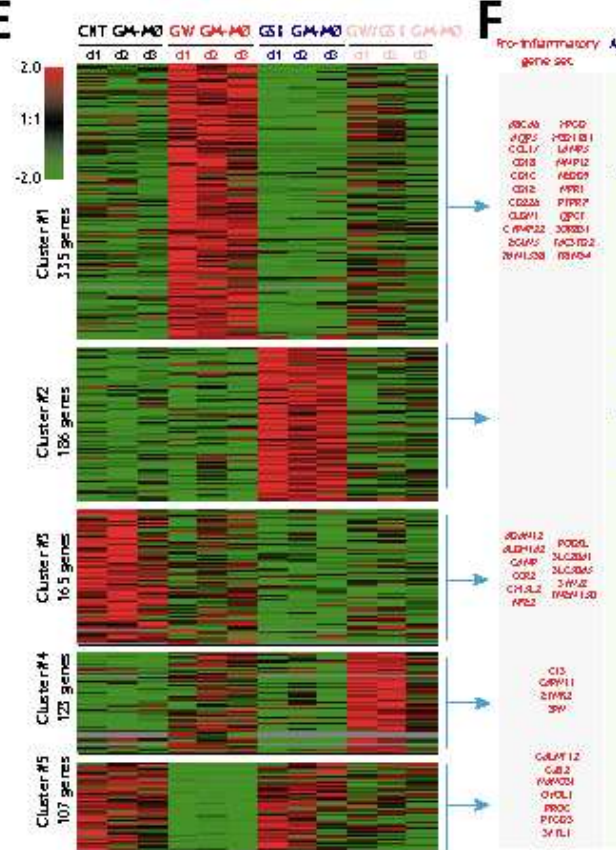

$\mathbf{F}$

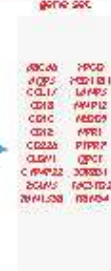

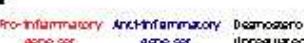

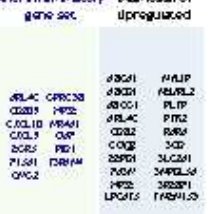

G

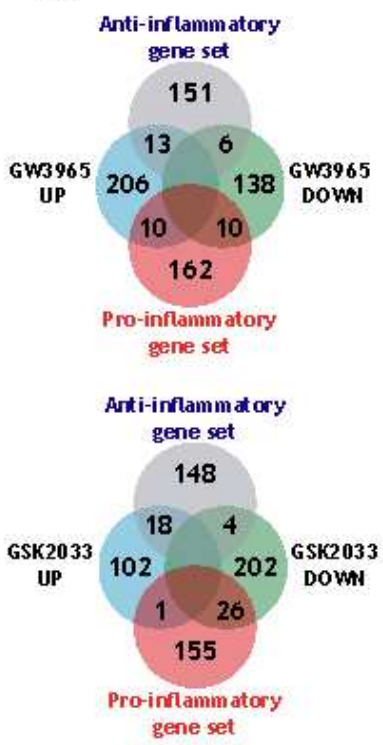

Figure 4

\section{Figure 4}

Identification of a novel set of LXR-dependent genes in proinflammatory GM-MØ. A. In vitro generation of control GM-MØ, GW3965-GM-MØ, GSK2033-GM-MØ and GSK2033+GW3965- GM-MØ before RNA isolation and RNA-sequencing. Control GM-M $\varnothing$ were exposed to DMSO in parallel. B. GSEA of desmosterol-upregulated genes in the ranked comparison GW3965-GM-M $\varnothing$ vs. GM-M $\varnothing$ and GSK2033GM-M $\varnothing$ vs. GM-M $\varnothing$. C. Number of differentially expressed genes ( $|\log 2 F C|>1$, adjp $<0.05$, ) relative to control GM-MØ. D. Summary of GSEA of the "Pro-inflammatory gene set" and "Anti-inflammatory gene set" on the ranked comparisons of the indicated transcriptomes. The color of the circles illustrates the type of enrichment of each comparison (positive, red; negative, blue). The area of each circle is 
proportional to the Normalized Enrichment Scores of each comparison, which is also indicated. Two representative GSEA profiles are shown at the top. E. Unsupervised clustering of differentially expressed genes $(|\log 2 \mathrm{FC}|>1)$ between control GM-M $\varnothing$ and the transcriptomes of GM-M $\varnothing$ generated in the presence of GW3965, GSK2033 or both. For each gene, mRNA expression level in the three donors are represented after normalizing gene expression and k-means clustering using Genesis

(http://genome.tugraz.at/genesisclient/). F. Indication of "Pro-inflammatory gene set", "Anti-inflammatory gene set" and desmosterol- upregulated genes in each cluster. G. Comparison of the "Pro-inflammatory gene set" and "Anti- inflammatory gene set" with the genes significantly regulated after GM-M $\varnothing$ differentiation in the presence of GW3965 (upper panel) or GSK2033 (lower panel).

A
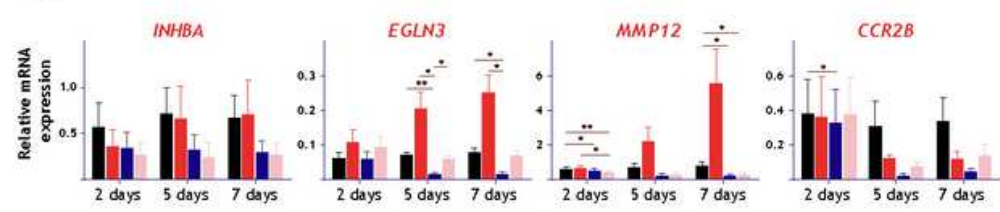

CD1B
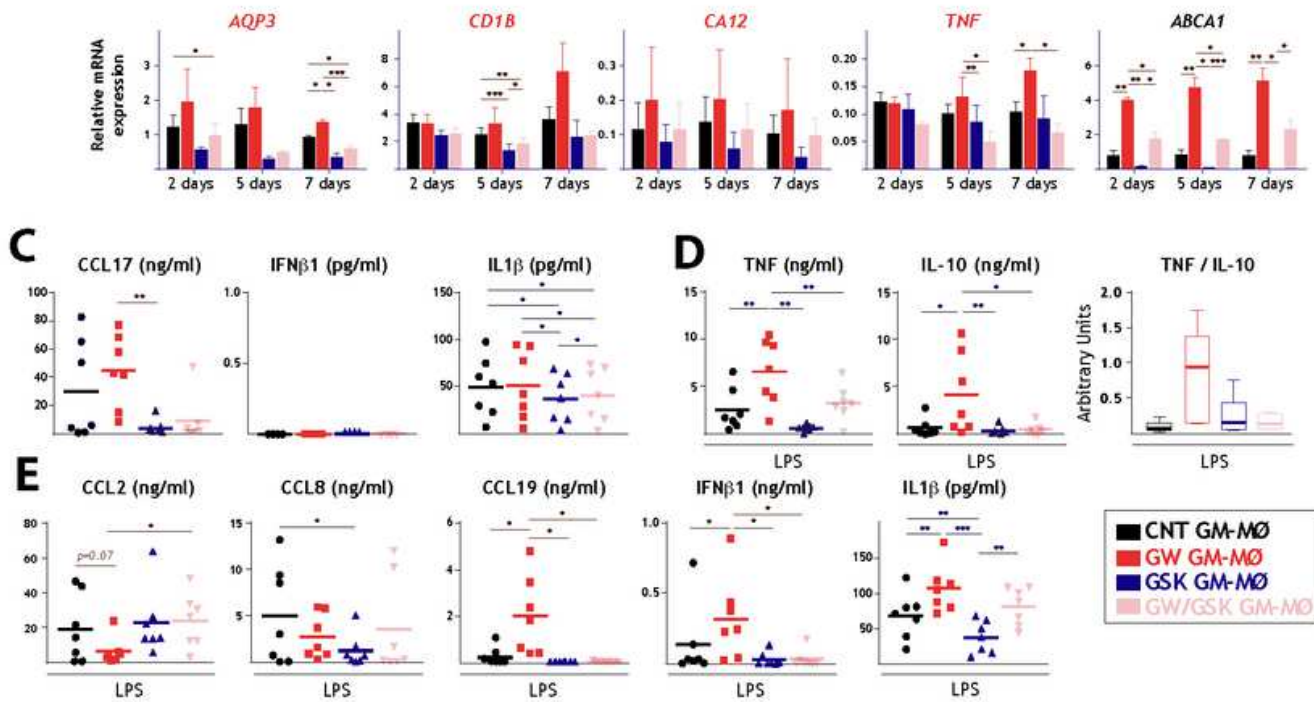
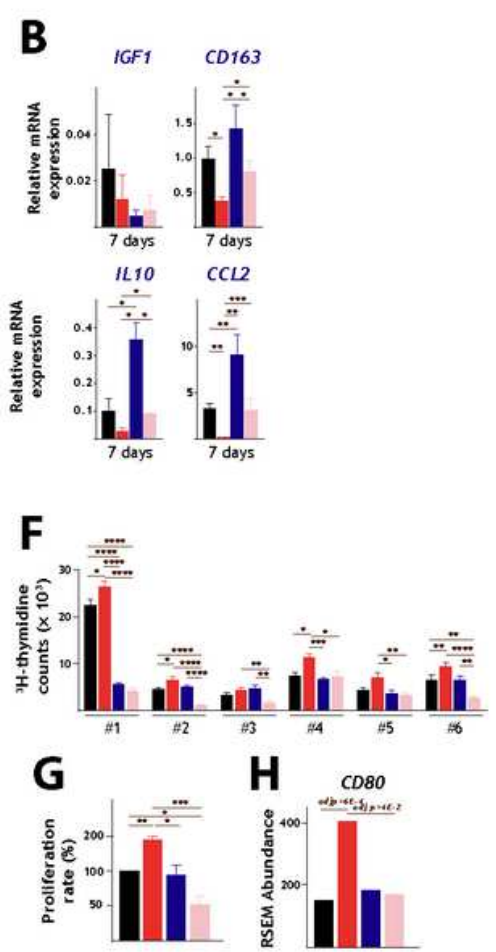

Figure 5

\section{Figure 5}

LXR activation in the generation of pro-inflammatory GM-MØ: time-dependency and functional consequences. A-B. Relative mRNA expression of the indicated genes from the "Pro- inflammatory" (A) and "Anti-inflammatory" $(B)$ gene sets in GM-M $\emptyset$ generated under the indicated treatments. ABCA1 expression was evaluated as a readout for LXR activation ( $\left.{ }^{*}, p<0.05 ; * \star, p<0.01 ; * \star *, p<0.001\right)$. C. Production of CCL17, IFN $₫ 1$ and IL-1区 in GM-M $\varnothing$ generated in the indicated conditions, as determined by ELISA. Mean \pm SEM of seven independent donors are shown $(*, p<0.05 ; * \star p<0.01)$. D. LPS-induced (10 $\mathrm{ng} / \mathrm{ml}, 18 \mathrm{~h}$ ) production of TNF and IL-10 in GM-MØ generated in the indicated conditions, as determined by ELISA. Mean \pm SEM of seven independent donors are shown $\left({ }^{*}, p<0.05 ; * *, p<0.01\right)$. The TNF/IL-10 is shown in the right panel. E. LPS-induced $(10 \mathrm{ng} / \mathrm{ml}, 18 \mathrm{~h})$ production of the indicated cytokines and chemokines in GM-M $\varnothing$ generated in the indicated conditions, as determined by ELISA. Mean \pm SEM of 
seven independent donors are shown $\left({ }^{*}, p<0.05 ; * \star, p<0.01 ; * \star *, p<0.001\right)$. F-G. T cell-stimulatory capacity of GM-MØ generated in the presence of GW3965 $(1 \mu \mathrm{M})$, GSK2033 $(1 \mu \mathrm{M})$ or both, as determined in Mixed Leukocyte Reactions (GM-M $\varnothing$ vs. allogeneic CD3+ T lymphocyte), and using GM-M $\varnothing$ generated in the presence of DMSO as a control (CNT GM-MØ). After a 6-day coculture, $3 \mathrm{H}$-thymidine incorporation was determined as a readout for $T$ cell proliferation. Mean \pm SEM of six independent experiments are shown. The relative T cell-stimulatory capacity (\% Proliferation rate) is shown in $G(*, p<0.05 ; * \star, p<0.01 ; * \star \star$, $\mathrm{p}<0.001 ; * \star \star \star, \mathrm{p}<0.0001)$. H. CD80 mRNA expression level in GM-M $\varnothing$ generated in the presence of GW3965 $(1 \mu \mathrm{M})$, GSK2033 $(1 \mu \mathrm{M})$ or both, as measured by the Expectation Maximization RNAseq (RSEM) algorithm, and using GM-M $\varnothing$ generated in the presence of DMSO as a control (CNT GM-M $\varnothing$ ). Adjusted $p$ values of indicated comparisons are shown.

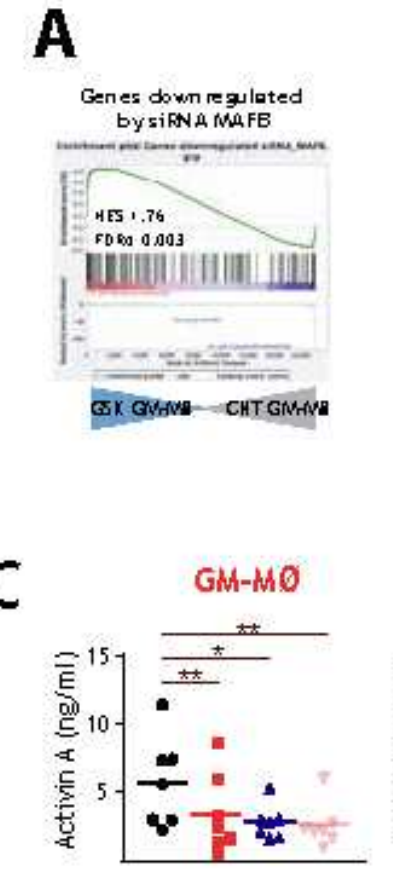

B
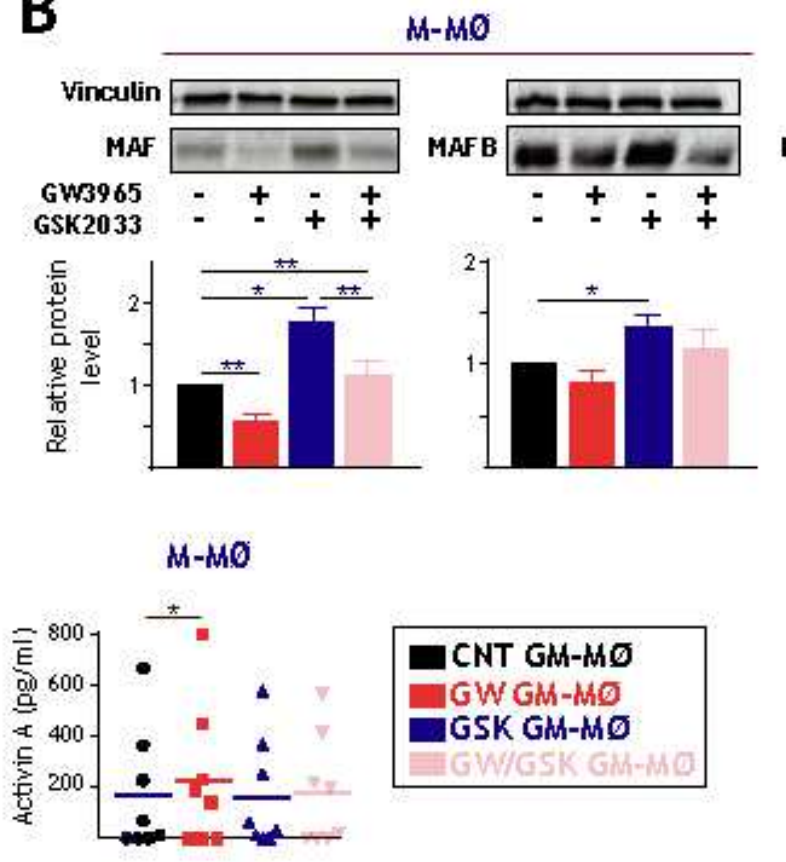

E

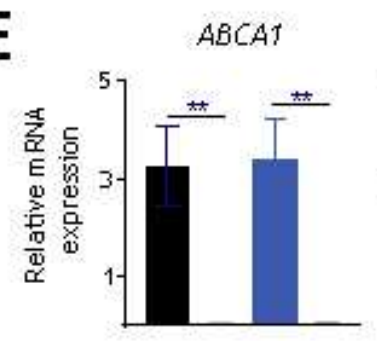

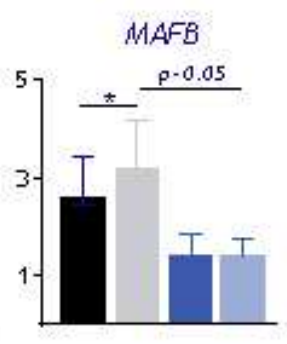
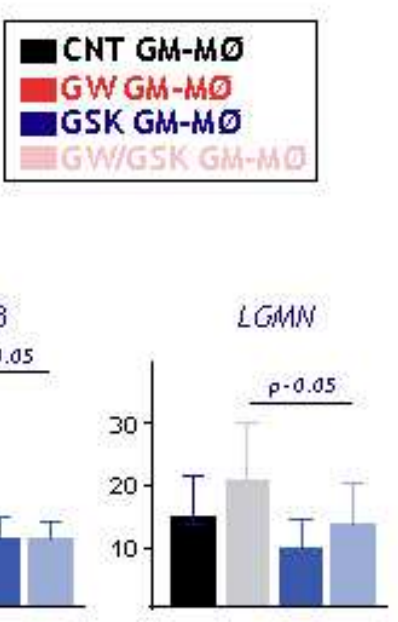

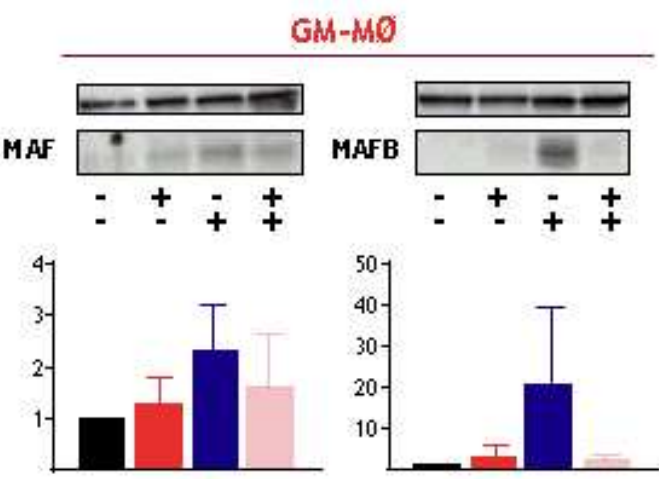

D

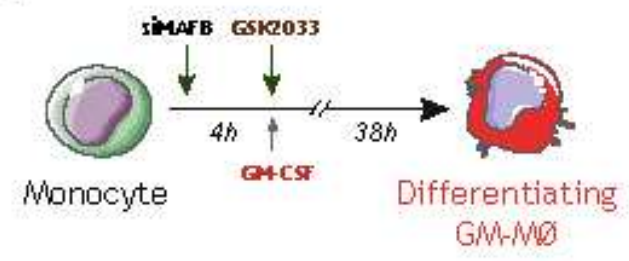

MARCKS

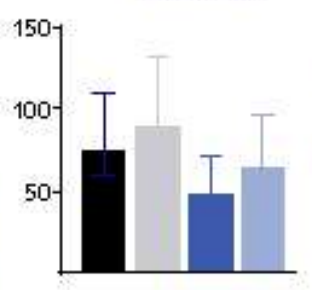

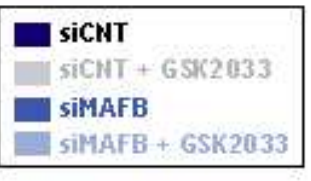

Figure 6

\section{Figure 6}

Molecular mechanisms underlying the macrophage polarizing effect of LXR. A. GSEA of genes downregulated by siRNA MAFB in the ranked comparison of the transcriptomes of GSK2033- GM-M $\varnothing$ and GM-M $\varnothing$. B. Relative protein levels of MAFB or MAF in M-MØ (left) and GM-M $\varnothing$ (right) generated in the presence of GW3965 $(1 \mu \mathrm{M})$, GSK2033 $(1 \mu \mathrm{M})$ or both, as determined by Western blot and densitometry, and using GM-M $\varnothing$ generated in the presence of DMSO as a control (insert). For protein loading control 
purpose, Vinculin protein levels for determined in parallel. Mean \pm SEM of the relative MAF and MAFB protein levels in M-M $\varnothing$ and GM-M $\varnothing$ from four independent donors are shown, and a representative Western blot experiment is shown in each case. C. Production of activin A in M-M $\varnothing$ and GM-MØ generated in the presence of the LXR agonist GW3965 $(1 \mu \mathrm{M})$, LXR inverse agonist GSK2033 $(1 \mu \mathrm{M})$ or both, as determined by ELISA, and using GM-M $\varnothing$ generated in the presence of DMSO as a control. Mean \pm SEM of seven (GM-M $\varnothing)$ or eight $(M-M \varnothing)$ independent donors are shown $(*, p<0.05 ; * \star, p<0.01)$. D.

Schematic representation of the assay to determine the effect of the LXR inverse agonist GSK2033 on the expression of MAFB and the MAFB target gene LGMN. E. Relative mRNA expression of the indicated genes in differentiating GM-M $\varnothing$ generated in the presence of GSK2033 and after siRNA-mediated knockdown of MAFB. Mean \pm SEM of seven independent experiments are shown $\left({ }^{*}, p<0.05 ;{ }^{* *} p<0.01\right)$.
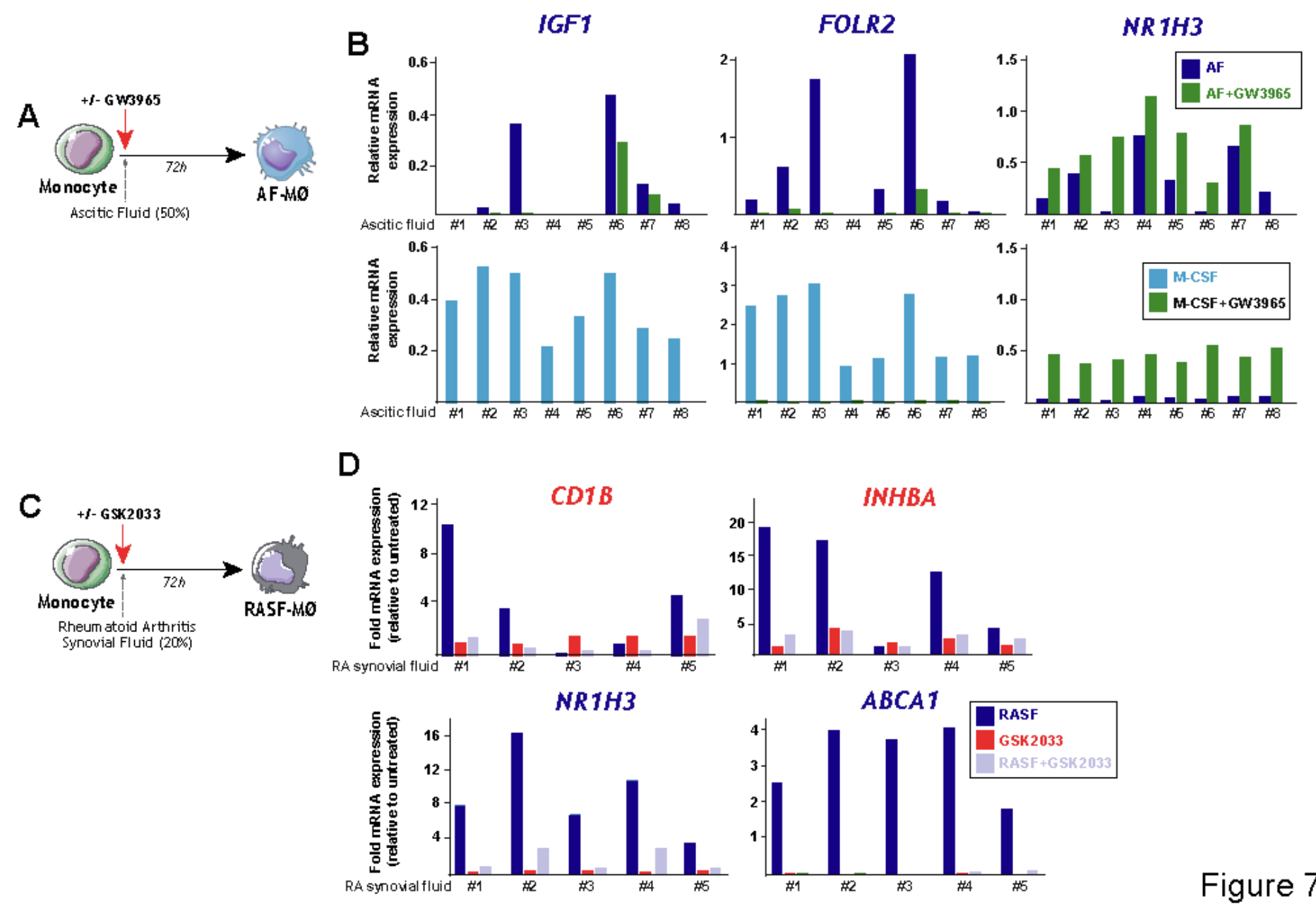

Figure 7

\section{Figure 7}

Physio-pathological relevance of the LXR-dependent expression of GM-M Ø-specific genes. A. Schematic representation for the generation of monocyte-derived macrophages in the presence of ascitic fluid from tumor patients (AF-M $\varnothing$ ). B. Relative mRNA expression of the indicated genes in M-M $\varnothing$ and AF-M $\varnothing$ generated in the absence of presence of GW3965. The results obtained with each individual ascitic fluid are shown. C. Schematic representation for the generation of monocyte-derived macrophages in the presence of Rheumatoid Arthritis synovial fluid (RASF-MØ). D. Expression of the indicated genes in RASF- 
$\mathrm{M} \varnothing$ generated in the presence or absence of GSK2033. Fold mRNA Expression indicates the expression of each gene relative to its expression in untreated monocytes kept in culture for 72 hours. The results obtained with each individual synovial fluid are shown.

\section{Supplementary Files}

This is a list of supplementary files associated with this preprint. Click to download.

- SupplementaryFigure1.pdf

- SupplementaryFigure2.pdf

- SupplementaryFigure3.pdf

- SupplementaryTablel.xlsx 